\title{
Synthetic Strategies and Parameters Involved in the Synthesis of Oligodeoxyribonucleotides According to the Phosphoramidite Method
}

The study of nucleic acids has, over the years, driven the development of fundamental methodologies necessary for the examination of their structure and chemistry. The ability to produce substantial quantities of sequence-defined synthetic DNA has been invaluable to nucleic acid research. Synthetic oligonucleotides have facilitated structural investigations and provided a better understanding of the interactions between nucleic acids and, for example, binding and/or modifying proteins. In addition, synthetic oligodeoxyribonucleotides have been extensively applied to the preparation of primers for enzymatic synthesis, amplification, and DNA sequencing. They have also been commonly used in site-directed mutagenesis experiments and as hybridization probes for diagnostic purposes. More recently, modified synthetic oligonucleotides have been targeted to cellular messenger RNAs in an attempt to control gene expression and develop therapeutic agents against various types of cancer and human infectious diseases (Beaucage and Iyer, 1993; Crooke and Bennett, 1996). On this basis, the availability of synthetic oligonucleotides has undoubtedly paved the way to the biotechnology revolution.

The basic strategy in oligonucleotide synthesis resembles that of the stepwise synthesis of polypeptides. Typically, a functionally protected nucleotide monomer is linked to a growing oligonucleotide chain that is then chemoselectively deprotected and allowed to couple with the next nucleotide monomer of the desired sequence. Although the coupling of nucleotide monomers was traditionally carried out in solution according to the phosphodiester (Khorana, 1968) and phosphotriester (Letsinger and Ogilvie, 1969) methods, these strategies ultimately culminated in the development of modern automated techniques using the phosphoramidite method (Beaucage and Caruthers, 1981; Caruthers et al., 1987a).

Given the major impact the phosphoramidite approach has had on the synthesis of oligonucleotides since its inception in the early 1980s, this commentary provides an overview of the parameters affecting the performance of the method and a number of compatible strategies for the preparation of oligodeoxyribonucleotides. Milestones that led to the discovery of the phosphoramidite method for oligodeoxyribonucleotide synthesis are chronologically reported. Alternate strategies to the preparation of deoxyribonucleoside phosphoramidites are then described to underscore the versatility with which these synthons can be obtained. The mechanisms of deoxyribonucleoside phosphoramidite activation and the factors affecting condensation rates are discussed along with the importance of "capping" and oxidation reactions toward defining optimal performance conditions for oligonucleotide synthesis. Finally, alternate phosphoramidite methods to oligodeoxyribonucleotide synthesis and additional deprotection strategies are presented to demonstrate facile access to synthetic oligonucleotides for a variety of applications. This unit is intended to complement APPENDIX $3 C$ on the synthesis and purification of oligonucleotides.

\section{ACCOUNTS OF CHEMICAL RESEARCH IN DNA OLIGONUCLEOTIDE SYNTHESIS}

A major advance in the chemical synthesis of oligodeoxyribonucleotides was accomplished in the mid-1970s through the development of the "phosphite triester" method (Letsinger and Lunsford, 1976). This approach relied on the use of P(III) chemistry and entailed the reaction of $5^{\prime}$-O-phenoxyacetyl thymidine S.1 with 2,2,2-trichloroethyl phosphorodichloridite S.2 to generate the corresponding deoxyribonucleoside-3'-O-phosphorochloridite $\mathbf{S . 3}$ and variable amounts of $\left(3^{\prime} \rightarrow 3^{\prime}\right)$-dinucleoside phosphite triester $\mathbf{S . 4}$ (Fig. 3.3.1). Addition of 3'-O-monomethoxytrityl thymidine to the reaction mixture gave, after treatment with aqueous iodine, the dinucleoside phosphate triester $\mathbf{S . 5}$ along with the symmetrical $\left(3^{\prime} \rightarrow 3^{\prime}\right)$ - and $\left(5^{\prime} \rightarrow 5^{\prime}\right)$ dinucleoside phosphate triesters $\mathbf{S . 6}$ and $\mathbf{S . 7}$, respectively. The formation of $\mathbf{S . 7}$ presumably resulted from the reaction of $3^{\prime}-O$ monomethoxytrityl thymidine with unreacted S.2. It should be noted that the phosphite triester 


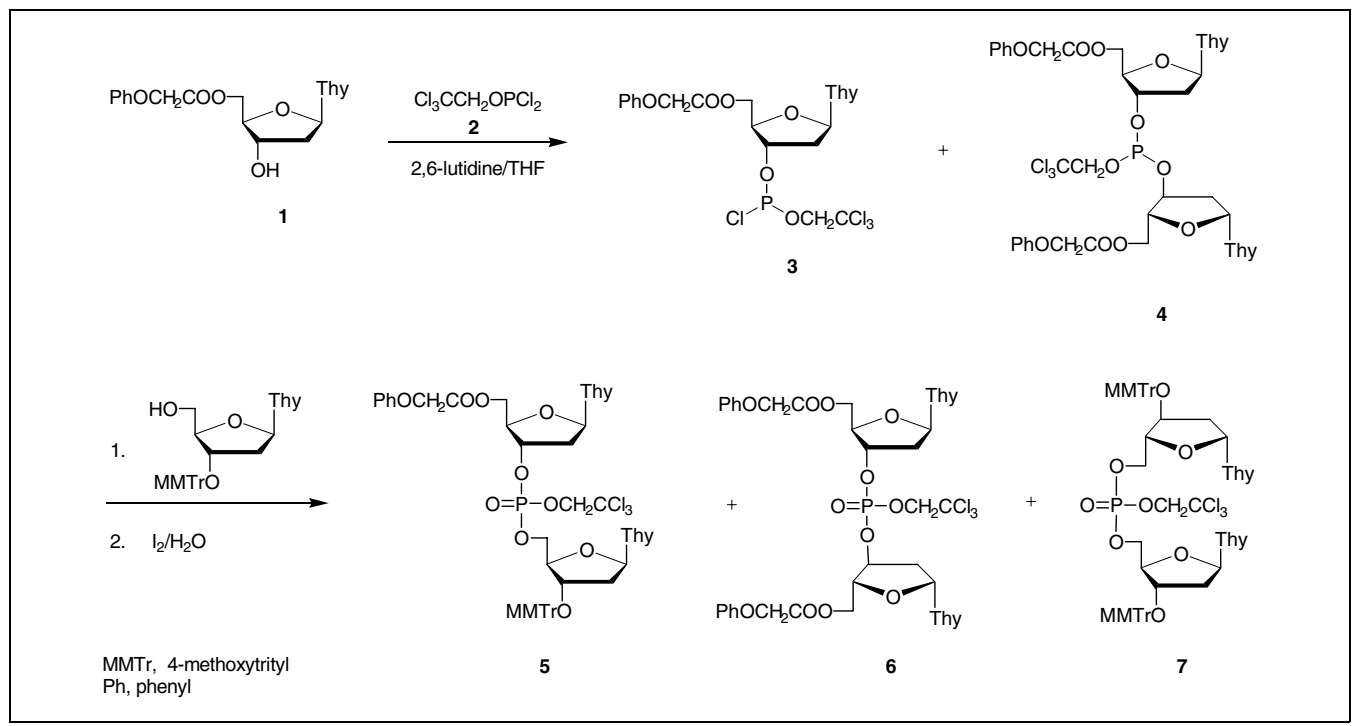

Figure 3.3.1 The phosphite triester method to oligodeoxyribonucleotide synthesis.

approach to oligonucleotide synthesis was remarkably rapid; the preparation of $\mathbf{S . 5}$ was complete within $1 \mathrm{hr}$. In the mid-1970s, the formation of internucleotide linkages with such kinetics was unmatched.

Later, the deoxyribonucleoside chlorophosphite S.8 (Fig. 3.3.2) was synthesized and reacted with deoxyribonucleosides covalently attached to a silica support (S.9) through a 3'-O-succinate linkage (Matteucci and Caruthers, 1981; see also UNITS 3.1 and 3.2). The dinucleoside phosphate triester $\mathbf{S . 1 0}$ was produced in yields $>90 \%$. Addition of $1 \mathrm{H}$-tetrazole to chlorophosphite $\mathbf{S . 8}$ significantly improved condensation rates and coupling yields. The application of nucleoside chlorophosphites or the corresponding tetrazolides to solid-phase synthesis of oligonucleotides was, however, tedious. Because of inherent sensitivity to moisture and air oxidation, the preparation of these reagents from reactive bifunctional phosphitylating agents had to be performed at $-78^{\circ} \mathrm{C}$ under rigorously anhydrous conditions and inert atmosphere. Although nucleoside chlorophosphites/tetrazolides led to the rapid and efficient preparation of DNA oligonucleotides on silica supports, the technical hardship associated with handling these synthons impeded automation of oligodeoxyribonucleotide syntheses.

These problems vanished when deoxyribonucleoside phosphoramidites were developed in the early 1980s (Beaucage and Caruthers, 1981). These synthons were stable to air oxidation and hydrolysis under normal laboratory conditions but readily activated under mild acidic conditions to form internucleotide linkages in near-quantitative yields. Because of these properties, deoxyribonucleoside phosphoramidites have been conducive to automated oligonucleotide synthesis on polymeric supports and are still the preferred synthons for such syntheses. The preparation of these reagents was straightforward and involved the reaction of protected deoxyribonucleosides, such as S.11, with chloro-( $N, N$-dimethy-

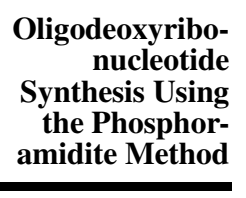

3.3.2

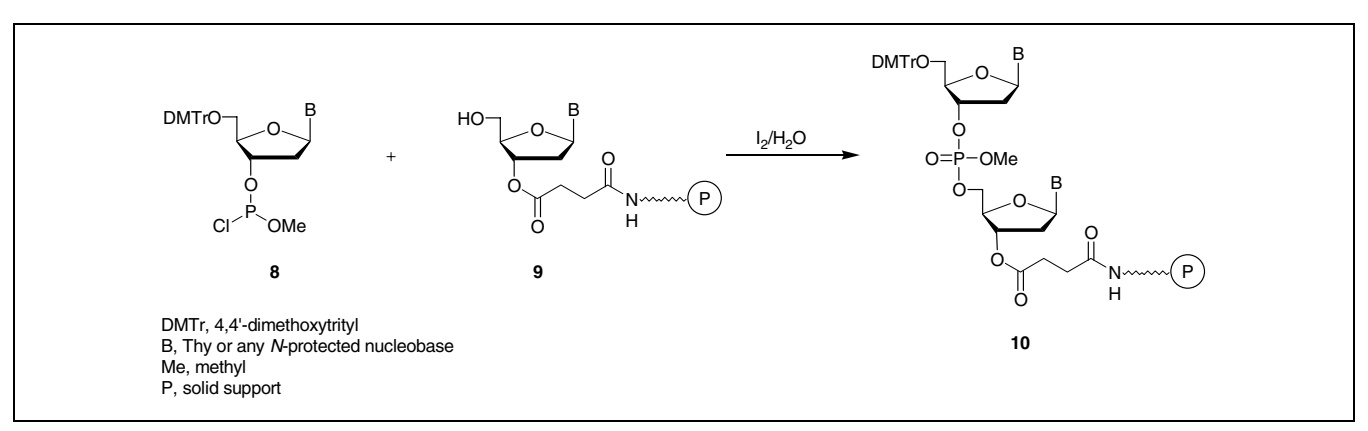

Figure 3.3.2 Application of the phosphite triester approach to solid-phase DNA synthesis. 


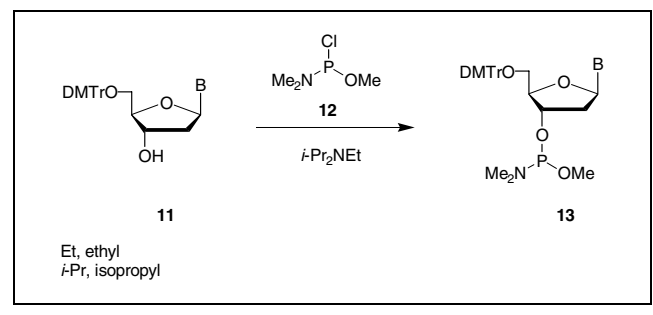

Figure 3.3.3 Preparation of deoxyribonucleoside phosphoramidite monomers.

lamino)methoxyphosphine $\mathbf{S . 1 2}$ and $N, N$-diisopropylethylamine (Fig. 3.3.3). The rapid reaction afforded deoxyribonucleoside phosphoramidites $\mathbf{S . 1 3}$ without the wasteful formation of $\left(3^{\prime} \rightarrow 3^{\prime}\right)$-dinucleoside phosphite triesters. The phosphoramidites $\mathbf{S . 1 3}$ were isolated by conventional laboratory techniques and stored as dry powders (Beaucage and Caruthers, 1981).

The phosphoramidite method is distinctive in that it enables the conversion of relatively stable deoxyribonucleoside phosphoramidite derivatives into highly reactive intermediates suitable for DNA oligonucleotide synthesis. For example, addition of $1 H$-tetrazole to the phosphoramidite $\mathbf{S . 1 3}$ and $3^{\prime}$ - $O$-levulinyl thymidine S.14 in dry acetonitrile generated the dinucleoside phosphite triester S.15 (Fig. 3.3.4) in almost quantitative yields within a few minutes, according to ${ }^{31} \mathrm{P}-\mathrm{NMR}$ spectroscopy (Beaucage and Caruthers, 1981). This methodology has been successfully applied to the solid-phase synthesis of oligodeoxyribonucleotides of varying chain lengths (Caruthers et al., 1982; Josephson et al., 1984). The sensitivity of phosphoramidite $\mathbf{S . 1 3}$ to a slightly acidic environment, however, precluded silica gel purification. Consequently, the chemical stability of crude S.13 in acetonitrile was variable, and the use of this type of phosphoramidite in automated systems not always reliable. Thus deoxyribonucleoside phosphoramidites with $\mathrm{N}, \mathrm{N}$-dialkylamino groups different from $\mathrm{N}, \mathrm{N}$ dimethylamino were investigated as potential alternatives to $\mathbf{S . 1 3}$ in automated solid-phase
DNA synthesis (Adams et al., 1983; McBride and Caruthers, 1983). For instance, the deoxyribonucleoside phosphoramidite S.16 (Fig. 3.3.5), unlike S.13, survived silica gel purification and, as a result, consistently showed stability in acetonitrile solutions for several days without significant decomposition (Adams et al., 1983). This class of phosphoramidites enabled reliable oligodeoxyribonucleotide syntheses (51-mers) on controlled-pore glass (CPG). In the early 1980s, these 51-mers were the largest DNA segments ever chemically synthesized.

One drawback to the use of S.16 in automated oligonucleotide synthesis is the noxious thiophenolate treatment required for postsynthesis removal of the methyl phosphate protecting groups (Daub and van Tamelen, 1977). Although demethylation of methyl phosphotriesters can be effected by 2-mercaptobenzothiazole (S.17; Andrus and Beaucage, 1988) or disodium 2-carbamoyl-2-cyanoethylene-1,1dithiolate (S.18; Dahl et al., 1990) under odorless conditions, this deprotection step was inconvenient because it added to the time and reagents needed for oligonucleotide processing (Fig. 3.3.5). In an effort to simplify and shorten postsynthesis deprotection protocols, the deoxyribonucleoside phosphoramidite S.19 (Sinha et al., 1984) was prepared under conditions similar to those originally reported by Beaucage and Caruthers (Fig. 3.3.5). This phosphoramidite derivative was more stable than the methyl phosphoramidite S.16 in wet acetonitrile (Zon et al., 1985). Furthermore, phosphoramidite S.19 generated oligonucleotides from which the 2-cyanoethyl phosphate protecting groups are cleaved under the basic conditions required for deprotection of the nucleobase protecting groups (Tener, 1961; Letsinger and Mahadevan, 1966; Letsinger and Olgilvie, 1969). Such a convenient "single step-single reagent" postsynthesis deprotection protocol led to the widespread acceptance of phosphoramidite $\mathbf{S . 1 9}$ for automated solidphase oligonucleotide synthesis.

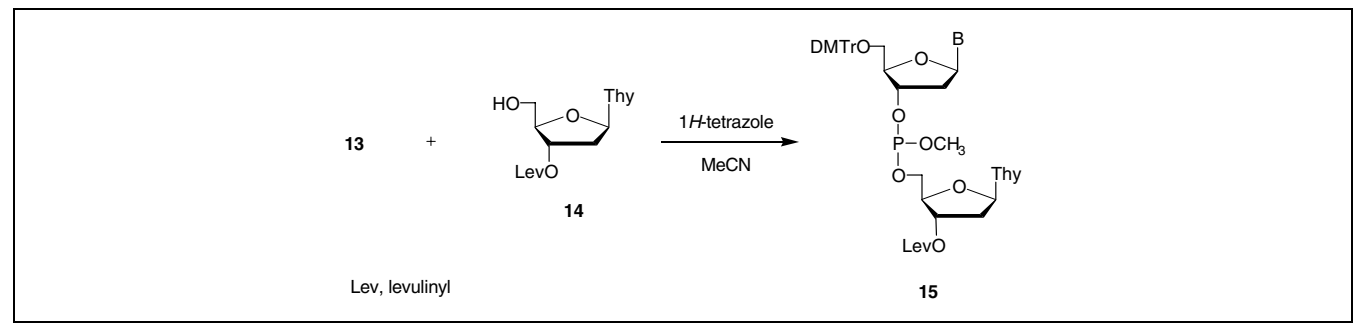

Figure 3.3.4 Activation of deoxyribonucleoside phosphoramidites toward oligonucleotide synthesis.

Synthesis of Unmodified Oligonucleotides

Current Protocols in Nucleic Acid Chemistry 


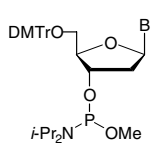

16

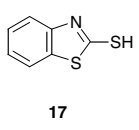

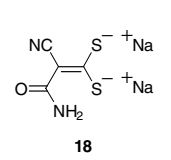

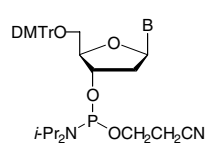

19

Figure 3.3.5 Deoxyribonucleoside phosphoramidite monomers with improved stability properties and reagents for the deprotection of methyl phosphate triesters.

\section{ALTERNATE STRATEGIES TO THE PREPARATION OF DEOXYRIBONUCLEOSIDE PHOSPHORAMIDITES}

The basicity and/or nucleophilicity of aminophosphine derivatives such as $\mathbf{S . 2 0}$ depends on the nature of the functional groups bound to phosphorus and on the interactions each of these groups might have with the vacant $d$ orbital of the phosphorus atom through $p \pi-d \pi$ overlap (Boudjebel et al., 1975). The bis-(dialkylamino) phosphine S.20 (Fig. 3.3.6) would, therefore, be more prone to protonation by a weak acid than would the deoxyribonucleoside phosphoramidite S.21, because it has been demonstrated that an alkoxy group contributes less to $p \pi-d \pi$ interactions than does a dialkylamino group (Mathis et al., 1974). This concept was convincingly tested when the deoxyribonucleoside $\mathbf{S . 1 1}$ was reacted with bis-(pyrrolidino)methoxyphosphine $\mathbf{S . 2 0}$ and the weak acid 4,5-dichloroimidazole (Beaucage, 1984; Moore and Beaucage, 1985) to generate the deoxyribonucleoside phosphoramidite S.21 within $10 \mathrm{~min}$, in yields exceeding 86\% (Fig. 3.3.6). Presumably because of weaker $p \pi-d \pi$ interactions, further activation of $\mathbf{S . 2 1}$ by $4,5-$ dichloroimidazole produced only a small amount of $\left(3^{\prime} \rightarrow 3^{\prime}\right)$-dinucleoside phosphite triester $(<10 \%)$. Thus, as soon as it was generated, phosphoramidite $\mathbf{S . 2 1}$ was immediately activated by addition of $1 \mathrm{H}$-tetrazole and used in the manual synthesis of an oligodeoxyribonucleotide (22-mer). This strategy eliminated the tedious isolation and purification of deoxyribonucleoside phosphoramidites and problems associated with the stability of phosphoramidite solutions. Despite these appealing features, this novel approach to the synthesis of deoxyribonucleoside phosphoramidites and oligonucleotides has never been automated, perhaps because of engineering limitations.

In this context, it has been shown that reaction of the deoxyribonucleoside $\mathbf{S . 1 1}$ with bis- $(\mathrm{N}, \mathrm{N}$ diisopropylamino)methoxyphosphine S.22 (Fig. 3.3.7) and limiting amounts of $1 H$-tetrazole or its $\mathrm{N}, \mathrm{N}$-diisopropylammonium salt afforded the corresponding deoxyribonucleoside phosphoramidite $\mathbf{S . 1 6}$ in isolated yields varying between $82 \%$ and 92\% (Barone et al., 1984; Lee and Moon, 1984). This method is definitely recommended for the preparation of a variety of 2-cyanoethyl deoxyribonucleoside phosphoramidite derivatives (like $\mathbf{S . 1 9}$ ) from phos-

\section{Oligodeoxyribo- nucleotide Synthesis Using the Phosphor- amidite Method}

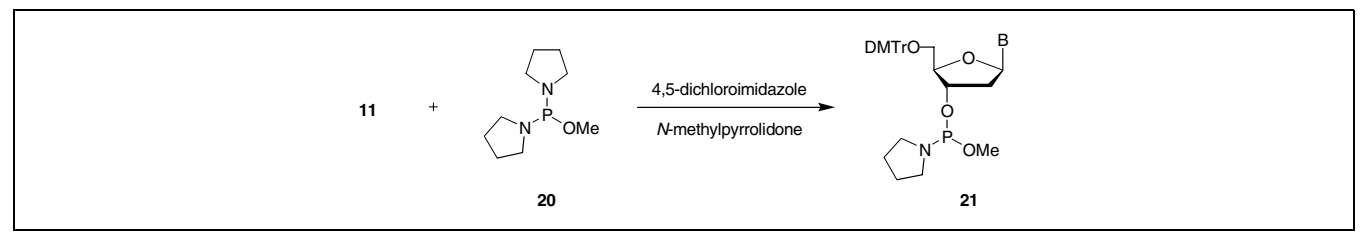

Figure 3.3.6 Chemoselective preparation of deoxyribonucleoside phosphoramidites in situ using bis-(pyrrolidino)methoxyphosphine and 4,5-dichloroimidazole.

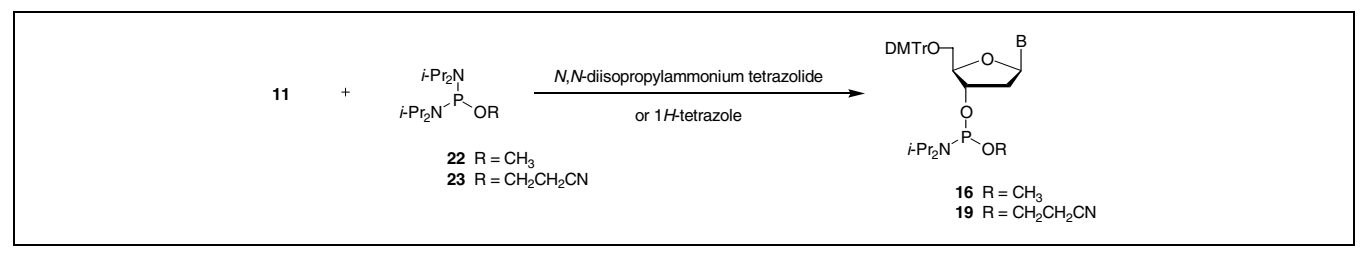

Figure 3.3.7 Chemoselective preparation of deoxyribonucleoside phosphoramidites using bis$(\mathrm{N}, \mathrm{N}$-diisopropyl)alkoxyphosphine and limiting amounts of $1 \mathrm{H}$-tetrazole or its $\mathrm{N}, \mathrm{N}$-diisopropylammonium salt. 


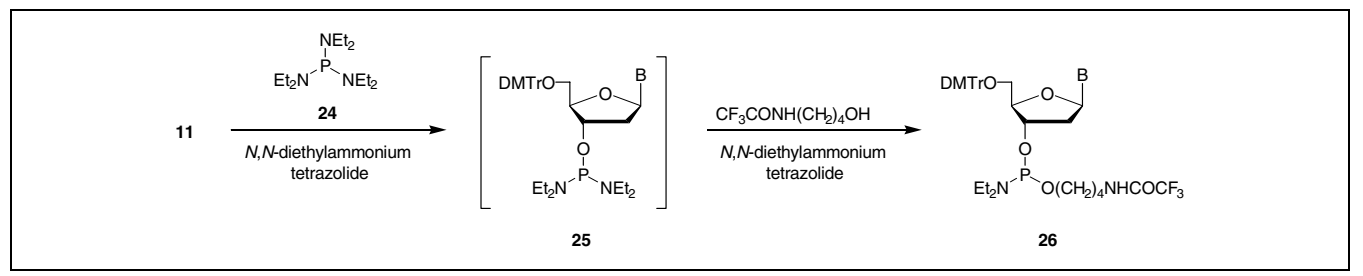

Figure 3.3.8 Preparation of deoxyribonucleoside phosphoramidites using hexaethylphosphorus triamide and limiting amounts of $\mathrm{N}, \mathrm{N}$-diethylammonium tetrazolide.

phorodiamidite $\mathbf{S . 2 3}$ (Fig. 3.3.7) because of the mildness and high chemoselectivity of the phosphinylation reaction.

Along similar lines, reaction of $\mathbf{S . 1 1}$ with hexaethylphosphorus triamide S.24 (Fig. 3.3.8) and an equimolar amount of the $N, N$-diethylammonium salt of $1 H$-tetrazole cleanly gave the nucleoside phosphorodiamidite $\mathbf{S . 2 5}$ (Yamana et al., 1989). Addition of, for example, 4-(N-trifluoroacetylamino)butan-1-ol to the reaction mixture produced the deoxyribonucleoside phosphoramidite S.26 (Fig. 3.3.8) in isolated yields exceeding 90\% (Wilk et al., 1997). The versatility of this procedure allows facile access to phosphoramidites different from the conventional methyl or 2-cyanoethyl deoxyribonucleoside phosphoramidites.

\section{ACTIVATION OF DEOXYRIBONUCLEOSIDE PHOSPHORAMIDITES}

The elegance of the phosphoramidite approach to oligodeoxyribonucleotide synthesis emanates from the transformation of relatively stable deoxyribonucleoside phosphoramidite derivatives to highly reactive intermediates for rapid and efficient chain extension reactions. Such a conversion is catalyzed by weak acids. For example, activation of phosphoramidite S.13 with $N, N$-dimethylaniline hydrochloride $\left(\mathrm{pK}_{\mathrm{a}} \approx 5.15\right)$ generated the deoxyribonucleoside chlorophosphite S.27 (Fig. 3.3.9) in quantitative yield according to ${ }^{31} \mathrm{P}-\mathrm{NMR}$ spectroscopy (Beaucage and Caruthers, 1981). Because most tertiary amine hydrochlorides are hygroscopic, these could not be used in routine

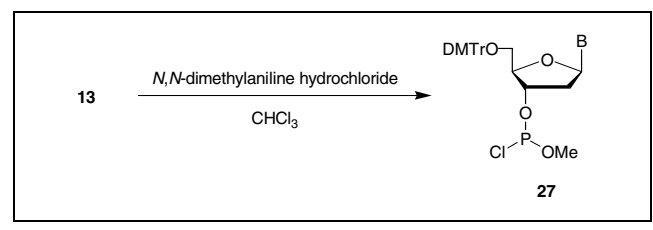

Figure 3.3.9 Activation of deoxyribonucleoside phosphoramidites with $\mathrm{N}, \mathrm{N}$-dimethylaniline hydrochloride. automated oligodeoxyribonucleotide synthesis because anhydrous conditions are absolutely necessary for optimum coupling reactions. Instead, the nonhygroscopic weak acid $1 H$-tetrazole has been and is still extensively used as an activator for deoxyribonucleoside phosphoramidites. In an incisive experiment, Seliger and Gupta (1985) demonstrated that activation of the solid-phase-linked deoxyribonucleoside phosphoramidite $\mathbf{S . 2 8}$ with $1 H$ tetrazole generated the putative phosphorotetrazolide derivative S.29 (Fig. 3.3.10). Immediate condensation of $\mathbf{S . 2 9}$ with a deoxyribonucleoside covalently attached to a solid support (such as S.9) gave the dinucleoside phosphite triester S.30 in yields greater than $95 \%$.

The generation of $\mathbf{S . 2 9}$ during activation of deoxyribonucleoside phosphoramidites by $1 \mathrm{H}$ tetrazole was further investigated using diethoxy(N,N-diisopropylamino)phosphine $\mathbf{S . 3 1}$ and diethoxy-( $N$-tetrazolyl)phosphine S.32 (Fig. 3.3.11) as models (Berner et al., 1989). ${ }^{31} \mathrm{P}-\mathrm{NMR}$ spectrum of $\mathbf{S . 3 2}$ shows a resonance at $126 \mathrm{ppm}$. This resonance is also apparent when $\mathbf{S . 3 1}$ or phosphoramidite $\mathbf{S . 1 6}$ is mixed with $1 H$-tetrazole in acetonitrile (McBride and Caruthers, 1983).

It should be noted that $P$-diastereomerically pure S.16 ( $S \mathrm{p}$ or $R \mathrm{p}$ ) rapidly epimerized at phosphorus upon activation with $1 H$-tetrazole and produced a diastereomeric deoxyribonucleoside phosphite triester when reacted with ethanol (Stec and Zon, 1984). These data indicate that activation of deoxyribonucleoside phosphoramidites with $1 H$-tetrazole occurs through a rapid and reversible protonation followed by a slower and reversible formation of the phosphorotetrazolide intermediate S.29. Paradoxically, activation of the nucleoside bicyclic phosphoramidites S.33-S.35 (Fig. 3.3.12) with $1 H$-tetrazole proceeded with low epimerization at phosphorus and led to highly stereoselective syntheses of oligonucleoside phosphorothioates (Guo et al., 1998; Iyer et al., 1998). A higher energy barrier to pseudorotation is apparently responsible for the reduced $P$-epimerization of these oxazaphospholidi-
Synthesis of Unmodified Oligonucleotides 


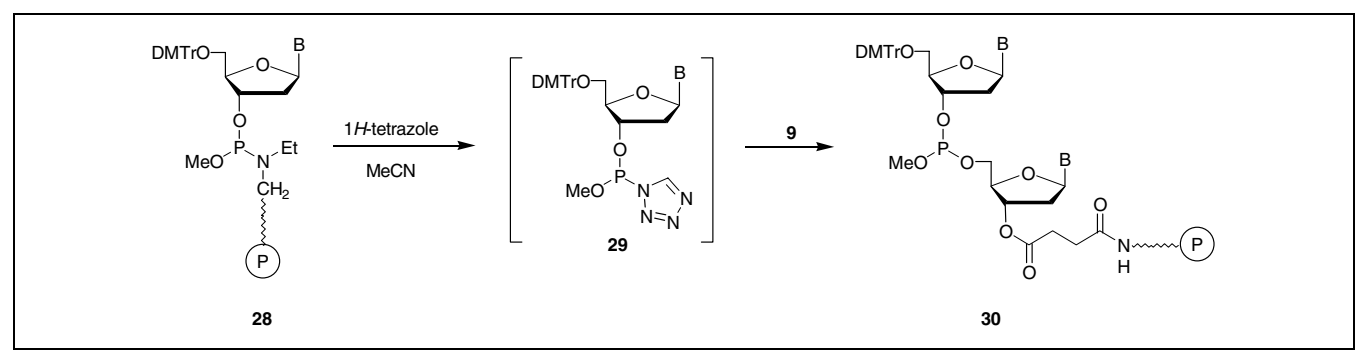

Figure 3.3.10 Mechanism of the activation of deoxyribonucleoside phosphoramidites by $1 \mathrm{H}$ tetrazole during solid-phase oligonucleotide synthesis.

nes during activation (Guo et al., 1998). It is somehow surprising that, although the conformationally restricted bicyclic oxazaphospholidines S.33 and S.34 allowed highly stereoselective syntheses of dinucleoside phosphorothioates $\left(S_{\mathrm{P}}: R_{\mathrm{P}}>9: 1\right)$, the bicyclic phosphoramidite $\mathbf{S . 3 6}$ produced the same dinucleotides with only moderate stereoselectivity $\left(R_{\mathrm{P}}: S_{\mathrm{P}} \approx 3: 1\right)$ (Guo et al., 1998). The bicyclic phosphoramidites S.33-S.35 are nonetheless promising candidates for the preparation of $P$-diastereomerically enriched oligonucleoside phosphorothioates.

Reagents other than $1 H$-tetrazole have also been used to activate deoxyribonucleoside phosphoramidites, including $\mathrm{N}$-methylimidazolium trifluoromethanesulfonate (Arnold et al., 1989); $N$-methylimidazole hydrochloride (Hering et al., 1985); pyridinium tetrafluoroborate (Brill et al., 1991); pyridinium chloride, pyridinium bromide, and pyridinium 4-methylbenzinesulfonate (Beier and Pfleiderer, 1999); $\mathrm{N}$-methylanilinium trifluoroacetate (Fourrey and Varenne, 1984); $N$-methylanilinium trichloroacetate (Fourrey et al., 1987); benzimidazolium triflate (Hayakawa et al., 1996); imidazolium triflate (Hayakawa and Kataoka, 1998); pyridine hydrochloride/imidazole (Gryaznov and Letsinger, 1992); 5-trifluoromethyl- $1 H$-tetrazole (Hering et al., 1985); 5-(4-nitrophenyl)-1 $H$-tetrazole (Froehler and Matteucci, 1983); 5-(2-nitrophenyl)$1 H$-tetrazole (Pon, 1987; Montserrat et al., 1994); 1-hydroxybenzotriazole (Claesen et al., 1984); 2,4,5-tribromo- and 2-nitro-imidazoles

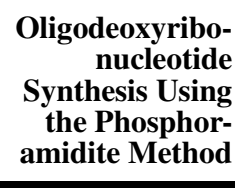

(Xin and Just, 1996); benzotriazole and 5-chlorobenzotriazoles (Xin and Just, 1996); and 4,5dichloro-, 2-bromo-4,5-dicyano-, and 4,5-dicyano-imidazoles (Xin and Just, 1996). 5Ethylthio- and 5-benzylthio- $1 H$-tetrazoles are also potent in the activation of phosphoramidites; these activators have been particularly useful in RNA synthesis (Wincott et al., 1995; Wu and Pitsch, 1998). It is important to note, however, that $1 H$-tetrazole and those more acidic activators $\left(\mathrm{pK}_{\mathrm{a}}<4.8\right)$ can cleave the 5'-dimethoxytrityl (DMTr) group of deoxyribonucleoside phosphoramidites to a small extent and trigger the formation of activated dimers. The coupling of these dimers during chain extension produced oligonucleotides longer $(n+1)$ than the expected size $(n)$ (Krotz et al., 1997a). The rates of DMTr deprotection by an activator depend on its acidity, exposure time, and nature of the nucleobase carrying the DMTr group (purines deprotect faster than pyrimidines). Typically, when the activation of deoxyribonucleoside phosphoramidites is performed by $1 H$-tetrazole for a period of $100 \mathrm{sec}$, $\sim 0.3 \%-0.9 \%$ of $(n+1)$-mers is observed; however, when $1 H$-tetrazole is replaced by 5 ethylthio- $1 H$-tetrazole under similar conditions, $\sim 1.0 \%-4.3 \%$ of $(n+1)$-mers are generated. It should, therefore, be understood that extended coupling times and the use of more acidic activators during solid-phase oligode-

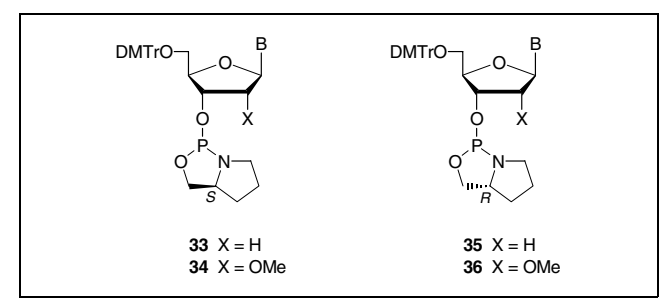

Figure 3.3.12 Nucleoside bicyclic phosphoramidites for the preparation of $P$-diastereomerically enriched oligonucleoside phosphorothioates. 
oxyribonucleotide synthesis will resultin lower recovery of full-length oligomers, because longer than full-length oligonucleotides will be produced. These observations prompted an extensive search for less-acidic, more-nucleophilic activators. It has been reported that 4,5-dicyanoimidazole (Xin and Just, 1996) is less acidic $\left(\mathrm{pK}_{\mathrm{a}}\right.$ 5.2), more soluble, and more nucleophilic than $1 H$-tetrazole (Vargeese et al., 1998). Moreover, the usefulness of 4,5-dicyanoimidazole relates not only to efficient synthesis of oligonucleotides but also to the preparation of nucleoside phosphoramidites from phosphorodiamidite S.23. Even though $1 \mathrm{H}$-tetrazole is still very popular as an activator for deoxyribonucleoside phosphoramidites, 4,5dicyanoimidazole is an attractive option for the activation of deoxyribonucleoside and ribonucleoside phosphoramites in solid-phase oligonucleotide synthesis.

\section{FACTORS AFFECTING THE CONDENSATION RATES OF DEOXYRIBONUCLEOSIDE PHOSPHORAMIDITES}

The steric bulk of specific guanine $N$-2 functional groups has been shown to affect significantly condensation rates and coupling efficiency of these deoxyribonucleoside phosphoramidite derivatives. Two classic examples illustrating this fact are the activation of phosphoramidites $\mathbf{S . 3 7}$ and $\mathbf{S . 3 8}$ with $1 H$-tetrazole (Fig. 3.3.13). In the case of activated S.37, a condensation time of 10 min generated cou- pling yields averaging $45 \%$ on a CPG support (Sekine et al., 1986). Under similar conditions, activated $\mathbf{S . 3 8}$ required a coupling time of 60 min to produce yields of $65 \%-70 \%$ (Casale and McLaughlin, 1990). Such coupling yields are far below those obtained with conventional deoxyribonucleoside phosphoramidites $(\sim 99 \%)$ and thus underscore the importance of steric hindrance when designing nucleobase protecting groups and/or modified nucleobases toward the synthesis of oligodeoxyribonucleotides and their analogues. One effective approach to lessening steric interferences is to increase the distance between the bulky entity and the phosphoramidite function by the use of flexible linkers. For example, unlike S.38, the deoxyribonucleoside phosphoramidite S.39 (Fig. 3.3.13) has been efficiently incorporated into oligonucleotides under the conditions used for standard 2-cyanoethyl deoxyribonucleoside phosphoramidites (Bergstrom and Gerry, 1994).

Other factors influencing the coupling rates of activated deoxyribonucleoside phosphoramidites have been investigated by Dahl and his colleagues (1987). In a systematic study, it was observed that condensation rates varied with the nature of phosphoramidite $O$ alkyl and $N, N$-dialkylamino groups. Typically, coupling rates decreased according to the following order: $O$-methyl $>O$-(2-cyanoethyl) $>$ $O$-(1-methyl-2-cyanoethyl) $>O$-(1,1-dimethyl-2-cyanoethyl) and $N, N$-diethylamino $>$

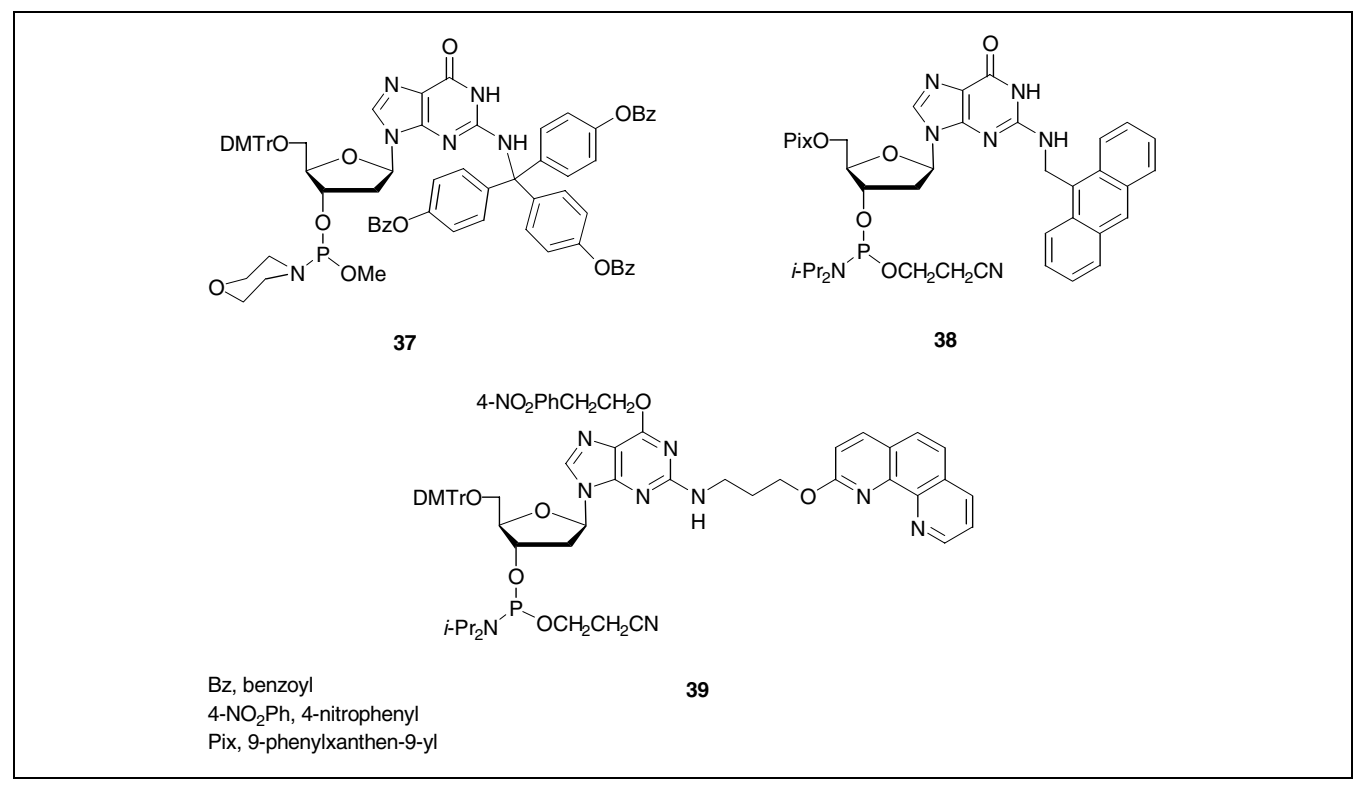

Figure 3.3.13 Deoxyribonucleoside phosphoramidites functionalized with nucleobase bulky groups. 
$\mathrm{N}, \mathrm{N}$-diisopropylamino $>\mathrm{N}$-morpholino $>\mathrm{N}$ methylanilino.

The effect of steric hindrance on coupling rates is further illustrated by activation of the 2 '-substituted nucleoside phosphoramidites S.40-S.43 (Fig. 3.3.14) and their competitive condensations with thymidine covalently attached to a solid support (Kierzek et al., 1987). Dimer formation was quantitated and correlated with the condensation rates of S.40-S.43. The amount of dimers formed decreased when the groups at $C-2^{\prime}$ increased in sizes; thus $2^{\prime}-\mathrm{H}$ $>2^{\prime}$ - $O$-methyl $>2^{\prime}$ - $O$-tetrahydropyranyl $>2^{\prime}$ $O$-tert-butyldimethylsilyl (Kierzek et al., 1987). In agreement with these findings, the deoxyribonucleoside phosphoramidites $\mathbf{S . 4 4}$ (Polushin, 1996) and S.45 (Jørgensen et al., 1994) have also exhibited significantly lower condensation rates and coupling efficiency because of steric factors (Fig. 3.3.14). Like sterically hindered groups attached to nucleobases (vide supra), it is also possible to decrease the steric demand of 2 - $O$-bulky protecting groups by increasing the distance between these groups and the phosphoramidite function. Specifically, the ribonucleoside $2^{\prime}$ - $O$-triisopropylsilyloxymethyl phosphoramidite $\mathbf{S . 4 6}$ (Weiss, 1998; Wu and Pitsch, 1998; Fig. 3.3.15) allows much faster coupling reactions (2 min) in solid-phase oligoribonucleotide synthesis than do ribonucleoside 2'-O-tertbutyldimethysilyl phosphoramidites $(5-8$ min) under essentially identical conditions (see UNIT 3.4). Similarly, coupling reactions of the ribonucleoside 2'-O-(o-nitrobenzyloxymethyl) phosphor- amidite S.47 (Fig. 3.3.15) are faster ( $2 \mathrm{~min})$ than those effected by ribonucleoside $2^{\prime}$ - $O$-(o-nitrobenzyl) phosphoramidites $(10 \mathrm{~min})$ under the same conditions (deBear et al., 1987; Schwartz et al., 1992). Thus functional groups generating

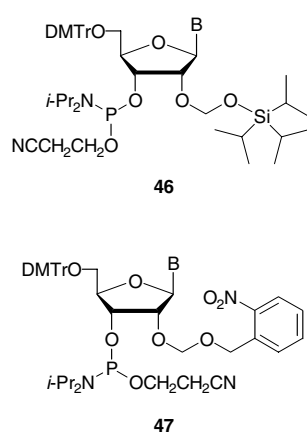

Figure 3.3.15 Efficient ribonucleoside phosphoramidites for solid-phase RNA synthesis.

steric bulk near the nucleosidic phosphoramidite moiety are likely to interfere with coupling rates and should be given consideration when developing novel phosphoramidite monomers.

\section{SIGNIFICANCE OF THE "CAPPING" REACTION IN THE CHEMICAL SYNTHESIS OF OLIGODEOXYRIBONUCLEOTIDES}

The phosphoramidite approach to oligodeoxyribonucleotide synthesis is renowned for its high coupling efficiency. Nonetheless, oligonucleotide chain extension does not occur quantitatively even under optimum conditions. As a result, the desired $n$-mer oligodeoxyribonucleotide is contaminated in the final product with a population of shorter $(n-1)$-oligomers. Separation of the $n$-mer oligonucleotide from $(n-1)$-mers can be challenging; however, this problem is almost completely eliminated by acetylation of the remaining unphosphitylated oligonucleotides after each condensation step. This "capping" reaction terminates the elongation of the unphosphitylated oligomers

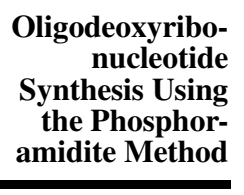

3.3.8

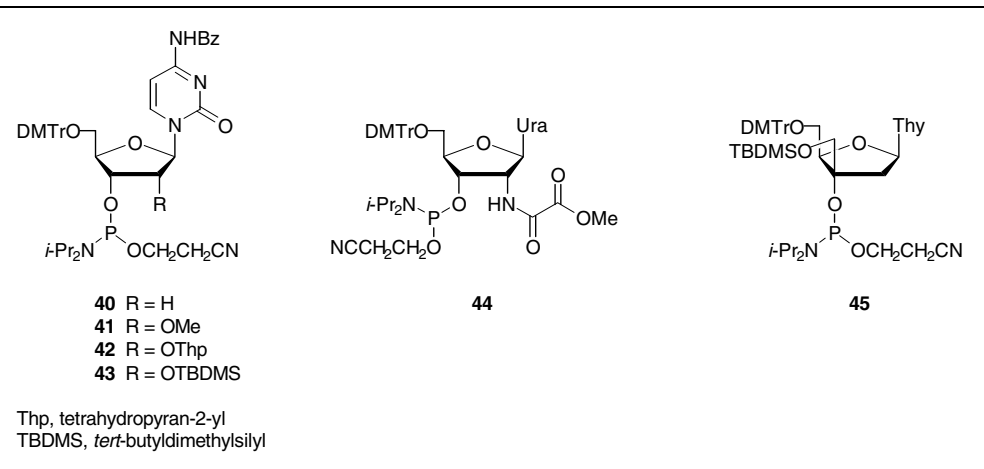

Figure 3.3.14 Nucleoside phosphoramidites functionalized with 2 '- or 3 '-sterically demanding groups. 
that would otherwise occur during the next coupling step. A very effective capping reagent is a solution of acetic anhydride, 2,6-lutidine, and $\mathrm{N}$-methylimidazole in tetrahydrofuran (Farrance et al., 1989). Such a capping formulation not only prevents extension of unphosphitylated oligomers but also efficiently reduces the concentration of $O^{6}$-phosphitylated guanine residues that are generated during the condensation step (Pon et al., 1986; Eadie and Davidson, 1987). The capping formulation is rich in acetate ions; these nucleophiles efficiently cleave $O^{6}$-phosphitylated guanine adducts by attacking tricoordinated "enol phosphites" (like S.48; Fig. 3.3.16) and releasing unmodified guanine residues. Adducts such as $\mathbf{S . 4 8}$ or its oxidized form $\mathbf{S . 4 9}$, if not destroyed, can serve as secondary sites for oligonucleotide synthesis and lead to the formation of a complex mixture of branched oligodeoxyribonucleotides. The generation of these adducts is most efficiently minimized when the capping reaction is performed before the oxidation step.

$O, O$-Diethyl- $N, N$-diisopropyl phosphoramidite has also been reported as an improved capping reagent in oligonucleotide synthesis (Yu et al., 1994). On the basis of the data presented, this phosphoramidite exhibited a capping efficiency that was only modestly superior to that of the standard acetic anhydride/ $N$-methylimidazole/2,6-lutidine capping formulation. The phosphoramidite capping reagent was also claimed to not produce nucleobase modification; supporting data were, however, not shown. Interestingly, recent use of the lipophilic $O$-(2-cyanoethyl), $O$-octyl- $N, N$-diisopropyl phosphoramidite as a capping re- agent in solid-phase oligonucleotide synthesis has allowed facile separation of capped failure sequences from trityl-off full-length oligonucleotides by reverse-phase HPLC (RP-HPLC; Natt and Häner, 1997). This capping method simplified RP-HPLC purification of synthetic oligonucleotides and resulted in higher isolated yields. Phosphoramidite capping reagents may, however, like nucleoside phosphoramidites, phosphitylate nucleobases (especially at $O-6$ of guanosines) and eventually lead to the formation of, for example, 2,6-diaminopurine residues (Eadie and Davidson, 1987). This potential problem has not, as yet, been thoroughly investigated. Until this issue is resolved, use of the standard and well-studied acetic anhydride/ $N$-methylimidazole/2,6-lutidine capping formulation during solid-phase oligonucleotide synthesis is recommended.

\section{THE OXIDATION REACTION IN THE SYNTHESIS OF OLIGODEOXYRIBONUCLEOTIDES ACCORDING TO THE PHOSPHORAMIDITE METHOD}

Oxidation of a newly generated phosphite triester linkage to the corresponding phosphate triester function is an essential step in automated synthesis of oligodeoxyribonucleotides by the phosphoramidite approach. Without oxidation, an internucleoside phosphite triester function decomposes under the acidic conditions required for cleavage of the $5^{\prime}$-dimethoxytrityl group (Matteucci and Caruthers, 1981). Thus oxidation of phosphite triesters is absolutely necessary to ensure consistent highyielding oligodeoxyribonucleotide syntheses. An aqueous solution of iodine $(0.05-0.1 \mathrm{M})$

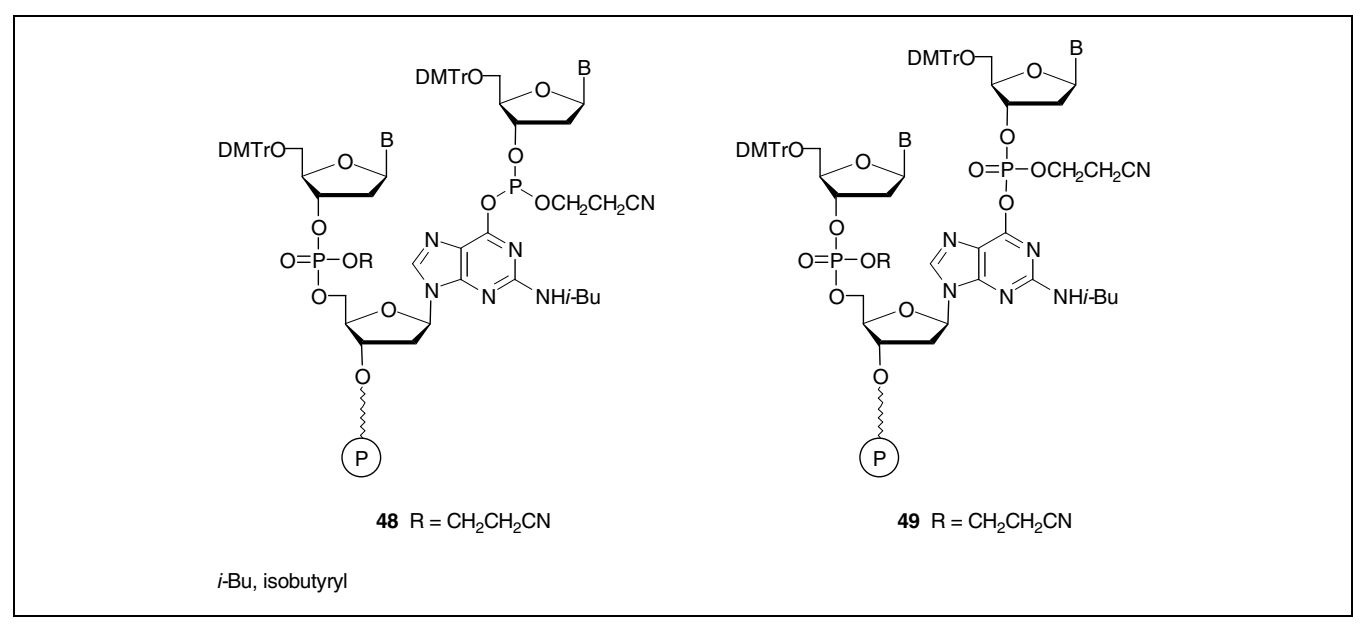

Figure 3.3.16 Postulated $O^{6}$-guanine adducts generated during the chain extension step of the synthesis cycle according to the phosphoramidite method.

Synthesis of Unmodified Oligonucleotides 
and 2,6-lutidine (Letsinger and Lunsford, 1976) or pyridine (Usman et al., 1985) in tetrahydrofuran is generally used for this task; this formulation is stable and provides rapid-reaction kinetics, usually without formation of side products. When the $\mathrm{N}-2$ amino function of guanine is protected with a $\mathrm{N}, \mathrm{N}$-dimethylformamidine group (Zemlicka and Holy, 1967; McBride et al., 1986; Vu et al., 1990) during automated oligonucleotide synthesis, however, use of the traditional iodine formulation as oxidant led to cyanation of guanine at $\mathrm{N}-2$ (Mullah et al., 1995). It has also been shown that this side reaction is completely eliminated by the use of a lower-concentration $(0.02 \mathrm{M})$ iodine oxidation reagent without losing speed and efficiency in the conversion of internucleoside phosphite triesters to phosphate triesters (Mullah et al., 1995). Thus the latter aqueous iodine formulation is recommended for standard oligonucleotide synthesis.

For specific applications, however, nonaqueous oxidizing reagents may advantageously offer an alternative to aqueous iodine for the oxidation of oligodeoxyribonucleoside phosphite triesters. For example, $m$-chloroperbenzoic acid (Tanaka and Letsinger, 1982); iodobenzene diacetate and tetra- $n$-butylammonium periodate (Fourrey and Varenne, 1985); tert-butyl hydroperoxide (Hayakawa et al., 1986; Hayakawa and Kataoka, 1998); ditert-butyl hydroperoxide; cumene hydroperoxide; hydrogen peroxide; bis-trimethylsilyl peroxide, and catalytic amounts of trimethylsilyl triflate (Hayakawa et al., 1986); dinitrogen tetroxide and molecular oxygen in the presence of 2,2'-azobis(2-methylpropionitrile) under thermal or photochemical conditions (Bentrude et al., 1989); and (1S)-(+)-(10-camphorsulfonyl) oxaziridine (S.50; Ugi et al., 1988) have been effective. The oxaziridine $\mathbf{S . 5 0}$ (Fig. 3.3.17) is particularly useful for the synthesis of oligonucleotide containing multiple 7 deaza- 2 '-deoxyguanosine residues. Incorpora-

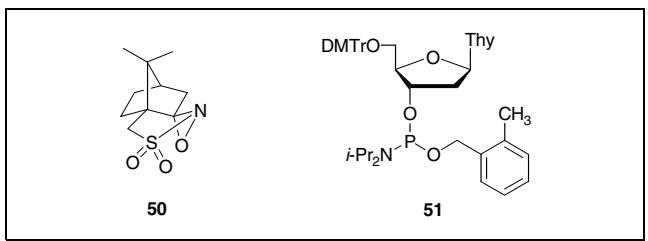

Figure 3.3.17 An oxaziridine derivative as a useful oxidant in the synthesis of oligonucleotides containing iodine sensitive residues, and a benzylic deoxyribonucleoside phosphoramidite suitable for the preparation of oligonucleotide analogues. tion of this modified 2'-deoxyguanosine into oligonucleotides via the phosphoramidite approach is sensitive to iodine-containing solutions regardless of iodine concentration (Anonymous, 1996).

In this context, it should be noted that when applied to oligonucleotide synthesis, the benzylic deoxyribonucleoside phosphoramidite S.51 (Fig. 3.3.17) generated internucleoside $o$-methylbenzyl phosphite esters that were sensitive to aqueous iodine oxidation. This sensitivity to iodine resulted in the loss of benzylic phosphate protection (Caruthers et al., 1987b). The absence of phosphate protecting groups did not, however, impair subsequent additions of S.51 to the DNA chain. In fact, an oligothymidylic acid (20-mer) was prepared by the iterative incorporation of $\mathbf{S . 5 1}$ with an average coupling efficiency of $96 \%$. It was speculated that phosphate-phosphite mixed anhydrides could have been generated from the interaction of phosphate diesters with activated $\mathbf{S . 5 1}$ and then cleaved by excess $1 H$-tetrazole to regenerate the deoxyribonucleoside phosphorotetrazolide intermediates needed for chain extension.

Because of the inherent hazards involved with handling peroxides, the use of oxaziridine S.50 is, therefore, recommended for the oxidation of phosphite triesters of those modified oligonucleotides that are reactive to iodine and/or necessitate rigorously anhydrous conditions. Moreover, the use of S.50 in oligonucleotide synthesis does not lead to detectable nucleobase modifications (Anonymous, 1996).

It has also been shown that oxidation of the dinucleoside 2-cyano-1,1-dimethylethyl phosphite triester S.52 (Fig. 3.3.18) with iodine in the presence of water, alcohols, and amines produced the corresponding dinucleoside phosphate S.54, phosphate triester S.55, and phosphoramidate S.56, respectively (Nielsen and Caruthers, 1988). It is postulated that under these oxidative Arbuzov-type conditions, elimination of the 2-cyano-1,1-dimethylethyl group led to the dinucleoside phosphoryl iodide intermediate S.53. The formation of $\mathbf{S . 5 3}$ is supported by ${ }^{31} \mathrm{P}-\mathrm{NMR}$ data and thus provides a versatile pathway to the synthesis of oligodeoxyribonucleotide analogues from deoxynucleoside 3'-O-(2-cyano-1,1-dimethylethyl) or $o$-methylbenzyl phosphoramidites.

Another example of the importance of P(III) oxidation in oligodeoxyribonucleotide synthesis according to the phosphoramidite approach is the incorporation of internucleotide phosphorothioates linkages into these bio- 


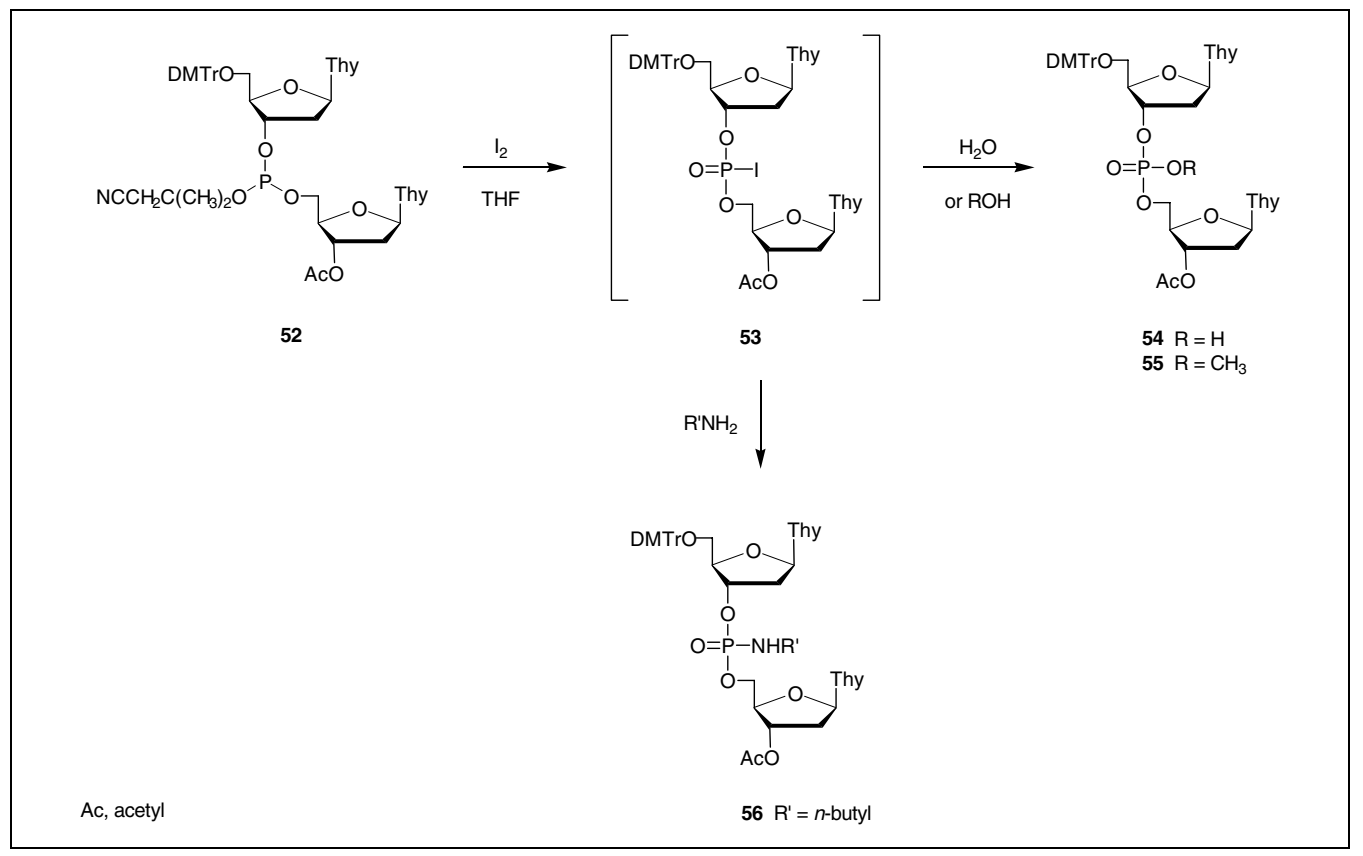

Figure 3.3.18 Access to oligodeoxyribonucleotide analogues from deoxynucleoside (2-cyano1,1-dimethylethyl) phosphoramidites.

molecules. Oligonucleotides carrying internucleotide phosphorothioate diesters display enhanced resistance to hydrolysis catalyzed by nucleases (Eckstein, 1985). Because of this property, oligodeoxyribonucleoside phosphorothioates have been extensively used as antisense molecules in the inhibition of gene expression. Automated synthesis of these modified oligonucleotides via the phosphoramidite method consists of replacing the aqueous iodine oxidation step by a sulfurization reaction that had originally been effected by elemental sulfur. Given the poor solubility of elemental sulfur in organic solvents, its use in automated systems has been difficult. This problem was eliminated when phenylacetyl disulfide (S.57; Kamer et al. 1989; Roelen et al., 1991) and 3H-1,2-benzodithiol-3-one 1,1-dioxide (S.58; Iyer et al., 1990; Regan et al., 1992) were employed as sulfurization reagents (Fig. 3.3.19). These compounds are soluble in organic solvents and produce efficient and rapid sulfurization kinetics. For example, $\mathbf{S . 5 8}$ converted the dinucleoside phosphite triester S.59 to the corresponding phosphorothioate dimer S.60 in yields exceeding 99\% within 30 sec at $25^{\circ} \mathrm{C}$ (Iyer et al., 1990; Regan et al., 1992). Deprotection of $\mathbf{S . 6 0}$ afforded the dinucleoside phosphorothioate S.61 (Fig. 3.3.19). Thus the sulfur-transfer reagent $\mathbf{S . 5 8}$ has enabled reliable automated synthesis of phosphorothioated oligomers carrying either exclusively or a predetermined number of phos- phorothioate groups (Iyer et al., 1990). Given the biological significance of oligonucleoside phosphorothioates, application of $\mathbf{S . 5 8}$ to the synthesis of these modified oligonucleotides has spurred interest in the development of additional sulfurizing reagents. The most notable sulfur-transfer agents that have been reported during this decade include $N, N, N^{\prime}, N^{\prime}$ tetraethylthiuram disulfide ( $\mathrm{Vu}$ and Hirschbein, 1991), dibenzoyl tetrasulfide (Rao et al., 1992), bis-(O,O-diisopropoxyphosphinothioyl) disulfide (Stec et al., 1993), benzyltriethylammonium tetrathiomolybdate (Rao and Macfarlane, 1994), bis(p-toluenesulfonyl)disulfide (Efimov et al., 1995), 3-ethoxy1,2,4-dithiazoline-5-one (Xu et al., 1996), thiiranes (Arterburn and Perry, 1997), bis(ethoxythiocarbonyl)tetrasulfide (Zhang et al., 1998), and 3-methyl-1,2,4-dithiazoline-5one (Zhang et al., 1999). Out of these sulfurtransfer reagents, $3 H$-1,2-benzodithiol-3-one 1,1-dioxide and 3-ethoxy-1,2,4-dithiazoline-5one are currently the most extensively used in solid-phase synthesis of oligonucleoside phosphorothioates.

\section{STRATEGIES IN THE DEPROTECTION OF SYNTHETIC OLIGODEOXYRIBONUCLEOTIDES}

The efficiency of the phosphoramidite method for solid-phase synthesis of oligodeoxyribonucleotides is such that oligonucleotides up to 50 bases long can be synthesized
Synthesis of Unmodified Oligonucleotides 


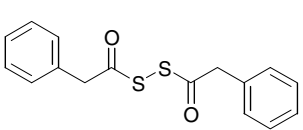

57

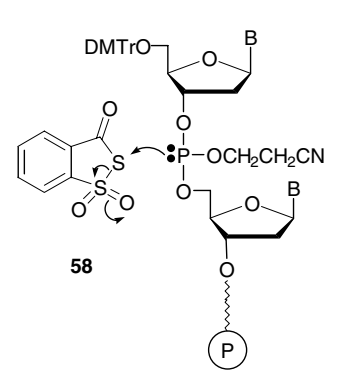

59

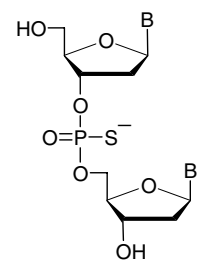

61

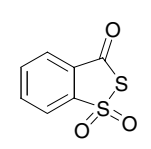

58

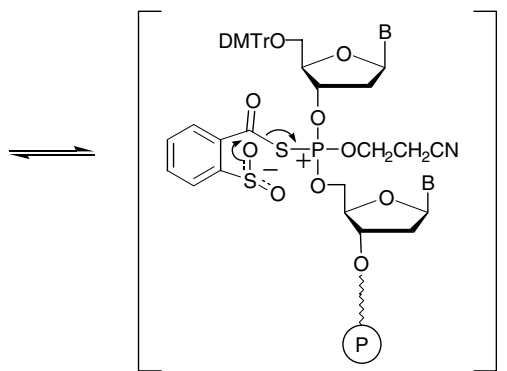

deprotection

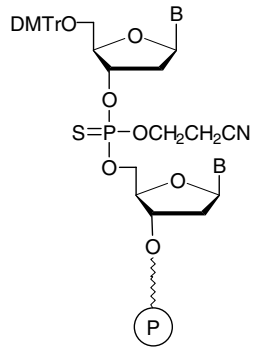

Figure 3.3.19 Preparation of oligodeoxyribonucleoside phosphorothioates according to the solidphase phosphoramidite method.

within a few hours. While the cleavage of these oligonucleotides from solid supports is normally accomplished by treatment with concentrated ammonium hydroxide for $\sim 1 \mathrm{hr}$ at ambient temperature, it will take $\sim 10 \mathrm{hr}$ at elevated temperature $\left(55^{\circ} \mathrm{C}\right)$ to deprotect the $N$-isobutyryl group of guanines, and $N$-benzoyl group of cytosines and adenines (see UNIT 2.1). This time-consuming deprotection step clashed with the urgent demand for synthetic oligodeoxyribonucleotides and thus provided an incentive to improve the chemistry involved with postsynthesis oligonucleotide processing. Specifically, methods for rapid removal of oligonucleotide protecting groups have attracted considerable attention and motivated the development of novel base-labile blocking groups for nucleobases (Schulhof et al., 1987; Uznanski et al., 1989; Kuijpers et al., 1990; Vu et al., 1990; Beaucage and Iyer, 1992; Sinha et al., 1993; Iyer et al., 1997; see also UNIT 2.1). Concentrated solutions of ammonia in water, ethanol, or methanol have been used for the cleavage of these groups. Alternatively, an aqueous solution of methylamine and ammonium hydroxide has been employed for the deprotection of oligonucleotides carrying $\mathrm{N}$-acetyl cytosines, $N$-benzoyl adenines, and $N$-isobutyryl guanines (Reddy et al., 1994). With this reagent, oligonucleotides were cleaved from solid supports in $5 \mathrm{~min}$ at ambient temperature, and complete deprotection was accomplished in $5 \mathrm{~min}$ at $65^{\circ} \mathrm{C}$. It should, however, be emphasized that an aqueous solution of methylamine and ammonium hydroxide cannot be used for the deprotection of oligonucleotides bearing conventional $N$-benzoyl cytosines because primary amines have been reported to attack $N^{4}$-anisoyl- or $N^{4}$-benzoyl-2'-deoxycytidine at $C$-4 to produce $N^{4}$-alkylated $2^{\prime}$-deoxycytidine derivatives (Weber and Khorana, 1972; Reddy et al., 1997).

Gaseous amines such as ammonia or methylamine have also been employed under pressure to achieve mild and rapid deprotection conditions (Boal et al., 1996). For example, oligodeoxyribonucleotides having cytosines, adenines, and guanines $N$-protected with a tert- 
butylphenoxyacetyl group were released from CPG supports and fully deprotected at $25^{\circ} \mathrm{C}$ by pressurized ammonia or methylamine within 35 or 2 min, respectively. It has also been shown that when the $N$-benzoyl group is used for protection of cytosines and adenosines, and $N$-isobutyryl for guanines, complete deprotection of oligodeoxyribonucleotides by ammonia gas will take $\sim 7 \mathrm{hr}$ at $25^{\circ} \mathrm{C}$. At that temperature, it would take $\sim 36 \mathrm{hr}$ for concentrated aqueous ammonium hydroxide to accomplish the same task (Boal et al., 1996).

The use of ammonia or methylamine gas allows the simultaneous deprotection of a large number of oligodeoxyribonucleotides. In fact, the number of oligonucleotides or CPG columns that can be deprotected is limited only by the size of the pressure vessel employed. Because no water is present during deprotection, fully deblocked oligonucleotides remain adsorbed to CPG and thus prevent cross-contamination between columns. Oligonucleotides can then be eluted from individual columns with a minimum amount of water for further purification, if desired. This deprotection procedure eliminates hazards inherent to the handling and heating of aqueous amine solutions in glass vials and, more important, the time-consuming evaporation of these solutions. The gas-phase deprotection methodology is recommended when oligonucleotides carrying base-sensitive nucleobases demand mild deprotection conditions or when rapid deprotection is needed to accelerate the production of synthetic oligonucleotides.

\section{ALTERNATE STRATEGIES TO THE SYNTHESIS OF OLIGODEOXYRIBONUCLEOTIDES ACCORDING TO THE PHOSPHORAMIDITE METHOD}

The versatility of the phosphoramidite approach to oligodeoxyribonucleotide synthesis has been further demonstrated by the use of deoxyribonucleoside phosphoramidites with unprotected nucleobases. The success of this strategy depends on a modified synthesis cycle protocol that involves treatment of the solid support with an equimolar solution $(0.1 \mathrm{M})$ of pyridine hydrochloride and aniline in acetonitrile (Gryaznov and Letsinger, 1991) or benzimidazolium triflate in methanol (Hayakawa and Kataoka, 1998) immediately after each condensation reaction. This treatment destroys nucleobase adducts that are forming on the oligonucleotidic chain during each coupling step. This procedure should facilitate the syn- thesis of oligonucleotides bearing base-sensitive functional groups because treatment with concentrated ammonium hydroxide at elevated temperature will no longer be required for oligonucleotide deprotection. Furthermore, depurination of adenine and guanine residues under the acidic conditions required for the removal of the $5^{\prime}$-O-DMTr group will become even less likely. More data are still needed to assess whether the synthesis of oligodeoxyribonucleotides according to the phosphoramidite method without nucleobase protection is trouble-free. The method is promising in that it may significantly expedite the production of synthetic oligonucleotides by shortening postsynthesis oligonucleotide processing time.

Another strategy toward the preparation of oligodeoxyribonucleotides entails the stepwise condensation of dinucleotide phosphoramidite blocks such as S.62-S.65 (Fig. 3.3.20) instead of conventional monomeric deoxyribonucleoside phosphoramidites for chain extension. Activation of $\mathbf{S . 6 2}$ with $1 H$-tetrazole produced coupling yields $(\sim 99 \%)$ similar to those generated by monomeric phosphoramidites (Kumar and Poonian, 1984). The incorporation of S.63 into oligonucleotides allowed syntheses of randomized DNA sequences containing the 20 codons corresponding to all natural amino acids (Neuner et al., 1998). The efficiency of dinucleotide phosphoramidites to solid-phase oligonucleotide synthesis has been further demonstrated by the preparation of a large oligomer (101-mer) through repetitive condensations of the dimeric phosphoramidite S.64 (Wolter et al., 1986). Furthermore, the impurity profile of oligonucleoside phosphorothioates synthesized by iterative coupling of the thioated dinucleotide phosphoramidite $\mathbf{S . 6 5}$ (Krotz et al., 1997b) showed at least 70\% reduction of the $(n-1)$-mers and a $\sim 50 \%$ reduction of phosphodiester formation when compared to profiles obtained by standard monomer phosphoramidite couplings.

The use of dimeric phosphoramidites in the synthesis of unmodified oligodeoxyribonucleotides has not been widely adopted, probably because a library of up to 16 combinatorial dimers had to be prepared to accomplish the synthesis of one oligonucleotide. Conversely, the application of dimeric phosphoramidites to oligonucleotide analogue synthesis has been popular especially for the incorporation of modified internucleotide bridges. For example, the dimeric 5'-phosphonate-linked thymidine phosphoramidite S.66 (Zhao and Caruthers, 1996) and S.67 (Kofoed and Caruthers, 1996)
Synthesis of Unmodified Oligonucleotides

Current Protocols in Nucleic Acid Chemistry 

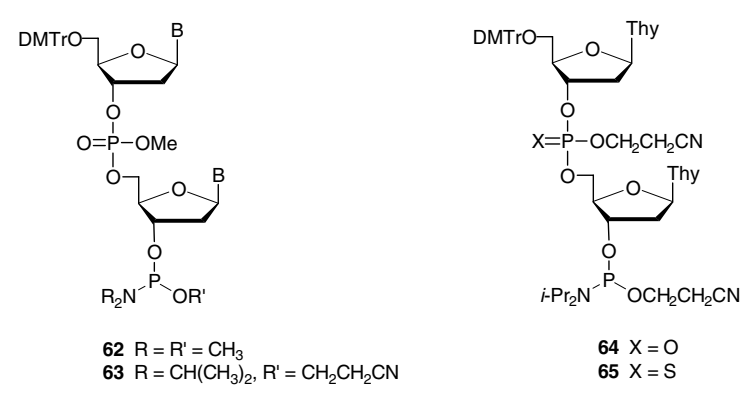

Figure 3.3.20 Solid-phase oligonucleotide synthesis using dinucleotide phosphoramidite derivatives.

have been prepared and incorporated into oligodeoxyribonucleotides to assess the physicochemical and biochemical properties imparted by such modifications (Fig. 3.3.21). For similar purposes, and given the growing interest in the development of therapeutic oligonucleotides, a plethoric number of dimeric phosphoramidites structurally related to $\mathbf{S . 6 6 - S . 6 8}$ have been prepared in recent years. Because of the intense activity in this area of research, only a fraction of the work has, so far, been reviewed (see Beaucage and Iyer, 1993; Sanghvi and Cook, 1994; Agrawal and Iyer, 1995; Iyer et al., 1999).

Oligodeoxyribonucleotides have also been prepared by the condensation of trinucleotide phosphoramidite blocks to enable oligonucleotide-directed mutagenesis. More and more, oligonucleotides of mixed composition are being used to generate combinatorial libraries of variants in the search for peptides and proteins with improved properties. The most direct route to controlled mutagenesis is indeed the use of trinucleotide synthons that correspond to the amino acid codons needed. The synthesis of trinucleotide phosphoramidites S.69 (Sondek and Shortle, 1992; Virnekäs et al., 1994), S.70 (Lyttle et al., 1995), S.71 (Ono et al., 1995; Kayushin et al., 1996; Zehl et al., 1996), and S.72 (Gaytán et al., 1998; Fig. 3.3.22) representing the codons for all 20 amino acids has been achieved. The incorporation of $\mathbf{S . 6 9}$ into oligonucleotides was accomplished by allowing a coupling time of $1 \mathrm{~min}$ and performing the trinucleotide condensation step twice. Under these conditions, coupling yields averaged 96\%-98.5\% (Virnekäs et al., 1994). Considering that each trinucleotide condensation adds three nucleobases to the growing oligonucleotide chain, these coupling yields are equivalent to three individual monomeric phosphoramidite condensations, each with a coupling efficiency of $98 \%-99.5 \%$. Incorporation of the trinucleotide phosphoramidites $\mathbf{S . 7 1}$ and S.72 into oligonucleotides via automated solidphase synthesis occurred in yields that varied with the sequence of the trinucleotide block used. Nonetheless, the incorporation of these trinucleotide phosphoramidite blocks into synthetic DNA in the controlled, codon-by-codon construction of combinatorial libraries of struc-

\section{Oligodeoxyribo- nucleotide Synthesis Using the Phosphor- amidite Method}

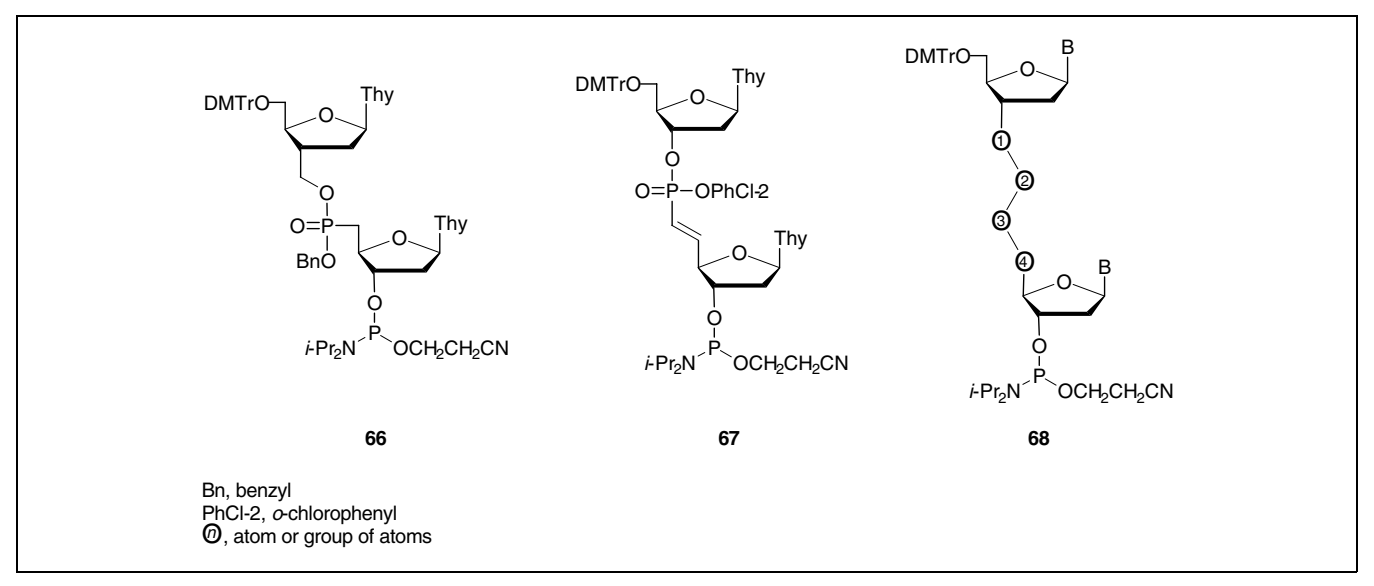

Figure 3.3.21 Solid-phase synthesis of oligonucleotide analogues from dimeric phosphoramidites carrying modified internucleotidic linkages. 
tural genes will be invaluable in creating molecular diversity by mutagenesis.

\section{CONCLUDING REMARKS}

Owing to the high performance of the phosphoramidite method, synthetic oligodeoxyribonucleotides became readily available and fueled the biotechnology revolution that has irreversibly changed biomedical research and the pharmaceutical industry. For example, without the ability to rapidly and efficiently synthesize DNA oligonucleotides, the development of the polymerase chain reaction (PCR) and its multiple applications would have been difficult, if not impossible, because this technology completely depends on the use of DNA primers. Similarly, the phosphoramidite method has been instrumental in the development of automated DNA sequencing, which also requires rapid and efficient synthesis of fluorescent DNA primers. Another important biological application for oligodeoxyribonucleotides generated by the phosphoramidite method relates to site-specific mutagenesis of protein genes. Mutagenesis of this type has been used to study protein structure-function relationships and to alter the therapeutic spectrum of pharmaceutically active proteins. In addition, the phosphoramidite method has been particularly useful in the synthesis of modified oligonucleotides for diagnostic applications and as potential therapeutic drugs. Although the latter research area is relatively new, several oligonucleotide-based drugs have already reached the clinic, and others are under preclinical investigation to benefit public health and push further the frontiers of knowledge.

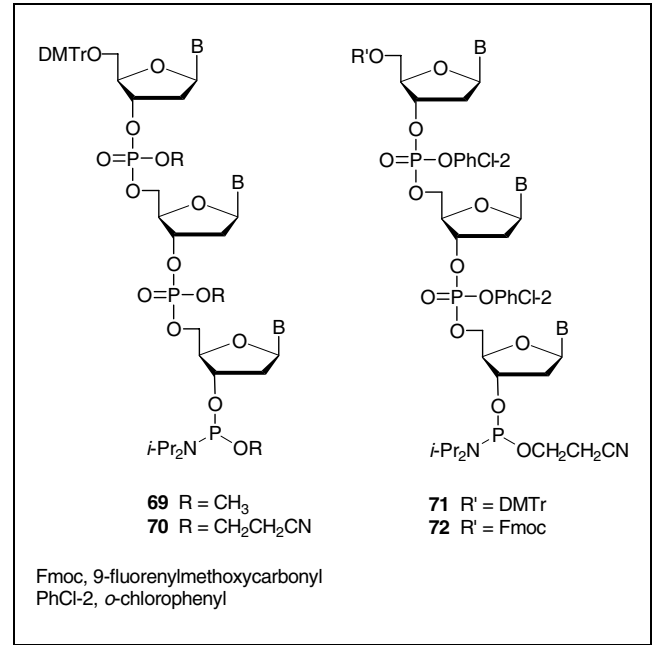

Figure 3.3.22 Trinucleotide phosphoramidite blocks for the controlled, codon-by-codon, construction of combinatorial gene libraries.
LITERATURE CITED

Adams, S.P., Kavka, K.S., Wykes, E.J., Holder, S.B., and Galluppi, G.R. 1983. Hindered dialkylamino nucleoside phosphite reagents in the synthesis of two DNA 51-mers. J. Am. Chem. Soc. 105:661663.

Agrawal, S. and Iyer, R.P. 1995. Modified oligonucleotides as therapeutic and diagnostic agents. Curr. Opin. Biotechnol. 6:12-19.

Andrus, A. and Beaucage, S.L. 1988. 2-Mercaptobenzothiazole-An improved reagent for the removal of methyl phosphate protecting groups from oligodeoxynucleotide phosphotriesters. Tetrahedron Lett. 29:5479-5482.

Anonymous. 1996. Non-aqueous oxidation with 10camphorsulfonyl-oxaziridine. The Glen Report 9:8-9.

Arnold, L., Tocik, Z., Bradkova, E., Hostomsky, Z., Paces, V., and Smrt, J. 1989. Automated chloridite and amidite synthesis of oligodeoxyribonucleotides on a long chain support using amidine protected purine nucleosides. Collect. Czech. Chem. Commun. 54:523-532.

Arterburn, J.B. and Perry, M.C. 1997. Rhenium catalyzed sulfurization of phosphorus(III) compounds with thiiranes: New reagents for phosphorothioate ester synthesis. Tetrahedron Lett. 38:7701-7704.

Barone, A.D., Tang, J.-Y., and Caruthers, M.H. 1984. In situ activation of bis-dialkylaminophosphines-A new method for synthesizing deoxyoligonucleotides on polymer supports. $\mathrm{Nucl}$. Acids Res. 12:4051-4061.

Beaucage, S.L. 1984. A simple and efficient preparation of deoxynucleoside phosphoramidites in situ. Tetrahedron Lett. 25:375-378.

Beaucage, S.L. and Caruthers, M.H. 1981. Deoxynucleoside phosphoramidites-A new class of key intermediates for deoxypolynucleotide synthesis. Tetrahedron Lett. 22:1859-1862.

Beaucage, S.L. and Iyer, R.P. 1992. Advances in the synthesis of oligonucleotides by the phosphoramidite approach. Tetrahedron 48:22232311.

Beaucage, S.L. and Iyer, R.P. 1993. The synthesis of modified oligonucleotides by the phosphoramidite approach and their applications. Tetrahedron 49:6123-6194.

Beier, M. and Pfleiderer, W. 1999. Pyridinium salts-An effective class of catalysts for oligonucleotide synthesis. Helv. Chim. Acta 82:879887.

Bentrude, W.G., Sopchik, A.E., and Gajda, T. 1989. Stereo- and regiochemistries of the oxidations of 2-methoxy-5-tert-butyl-1,3,2-dioxaphosphori nanes and the cyclic methyl $3^{\prime}, 5^{\prime}$-phosphite of thymidine by $\mathrm{H}_{2} \mathrm{O} / \mathrm{I}_{2}$ and $\mathrm{O}_{2} / \mathrm{AIBN}$ to P-chiral phosphates. ${ }^{17} \mathrm{O}$ NMR assignment of phosphorus configuration to the diastereomeric thymidine cyclic methyl 3',5'-monophosphates. J. Am. Chem. Soc. 111:3981-3987.
Synthesis of Unmodified Oligonucleotides 
Bergstrom, D.E. and Gerry, N. 1994. Precision sequence-specific cleavage of a nucleic acid by a minor-groove-directed metal-binding ligand linked through N-2 of deoxyguanosine. J. Am. Chem. Soc. 116:12067-12068.

Berner, S., Mühlegger, K., and Seliger, H. 1989. Studies on the role of tetrazole in the activation of phosphoramidites. Nucl. Acids Res. 17:853864.

Boal, J.H., Wilk, A., Harindranath, N., Max, E.E., Kempe, T., and Beaucage, S.L. 1996. Cleavage of oligodeoxyribonucleotides from controlledpore glass supports and their rapid deprotection by gaseous amines. Nucl. Acids Res. 24:31153117.

Boudjebel, H., Gonçalves, H., and Mathis, F. 1975. Étude de la liaison $\mathrm{P}-\mathrm{N}$ dans le motif $\mathrm{S}_{2} \mathrm{P}-$ $\mathrm{NMe}_{3}$ en résonance magnétique nucléaire et par la réaction d'échange avec le trifluoroacétate de méthyle. Bull. Chem. Soc. Chim. France 628634.

Brill, W.K.-D., Nielsen, J., and Caruthers, M.H. 1991. Synthesis of deoxydinucleoside phosphorodithioates. J. Am. Chem. Soc. 113:39723980.

Caruthers, M.H., Beaucage, S.L., Becker, C., Efcavitch, W., Fisher, E.F., Galluppi, G., Goldman, R., deHaseth, P., Martin, F., Matteucci, M., and Stabinsky, Y. 1982. New methods for synthesizing deoxyoligonucleotides. In Genetic Engineering: Principles and Methods, Vol. 4 (J.K. Setlow and A. Hollaender, eds.) pp. 1-17. Plenum, New York.

Caruthers, M.H., Barone, A.D., Beaucage, S.L., Dodds, D.R., Fisher, E.F., McBride, L.J., Matteucci, M., Stabinsky, Z., and Tang, Y.-Y. 1987a. Chemical synthesis of deoxyoligonucleotides by the phosphoramidite method. In Methods and Enzymology; Vol. 154 (R. Wu and L. Grossman, eds.) pp. 287-313. Academic Press, San Diego; and references therein.

Caruthers, M.H., Kierzek, R., and Tang, J.Y. 1987b. Synthesis of oligonucleotides using the phosphoramidite method. In Biophosphates and Their Analogues-Synthesis, Structure, Metabolism and Activity (K.S. Bruzik and W.J. Stec, eds.) pp. 3-21. Elsevier/North Holland, Amsterdam.

Casale, R. and McLaughlin, L.W. 1990. Synthesis and properties of an oligodeoxynucleotide containing a polycyclic aromatic hydrocarbon site specifically bound to the $\mathrm{N}^{2}$ amino group of a $2^{\prime}$-deoxyguanosine residue. J. Am. Chem. Soc. 112:5264-5271.

Claesen, C., Tesser, G.I., Dreef, C.E., Marugg, J.E., van der Marel, G.A., and van Boom, J.H. 1984. Use of 2-methylsulfonylethyl as a phosphorus protecting group in oligonucleotide synthesis via a phosphite triester approach. Tetrahedron Lett. 25:1307-1310.

Crooke, S.T. and Bennett, C.F. 1996. Progress in antisense oligonucleotide therapeutics. Аnпи. Rev. Pharmacol. Toxicol. 36:107-129.
Dahl, B.H., Nielsen, J., and Dahl, O. 1987. Mechanistic studies on the phosphoramidite coupling reaction in oligonucleotide synthesis. I. Evidence for nucleophilic catalysis by tetrazole and rate variations with the phosphorus substituents. Nucl. Acids Res. 15:1729-1743.

Dahl, B.H., Bjergårde, K., Henriksen, L., and Dahl, O. 1990. A highly reactive, odourless substitute for thiophenol/triethylamine as a deprotection reagent in the synthesis of oligonucleotides and their analogues. Acta Chem. Scand. 44:639-641.

Daub, G.W. and van Tamelen, E.E. 1977. Synthesis of oligoribonucleotides based on the facile cleavage of methyl phosphotriester intermediates. $J$. Am. Chem. Soc. 99:3526-3528.

deBear, J.S., Hayes, J.A., Koleck, M.P., and Gough, G.R. 1987. A universal glass support for oligonucleotide synthesis. Nucleosides Nucleotides 6:821-830.

Eadie, J.S. and Davidson, D.S. 1987. Guanine modification during chemical DNA synthesis. Nucl. Acids Res. 15:8333-8349.

Eckstein, F. 1985. Nucleoside phosphorothioates. Annu. Rev. Biochem. 54:367-402.

Efimov, V.A., Kalinkina, A.L., Chakhmakhcheva, O.G., Schmaltz Hill, T. and Jayaraman, K. 1995. New efficient sulfurizing reagents for the preparation of oligodeoxyribonucleotide phosphorothioate analogues. Nucl. Acids Res. 23:4029-4033.

Farrance, I.K., Eadie, J.S., and Ivarie, R. 1989. Improved chemistry for oligodeoxyribonucleotide synthesis substantially improves restriction enzyme cleavage of a synthetic 35 mer. Nucl. Acids Res. 17:1231-1245.

Fourrey, J.-L. and Varenne, J. 1984. Improved procedure for the preparation of deoxynucleoside phosphoramidites: Arylphosphoramidites as new convenient intermediates for oligodeoxynucleotide synthesis. Tetrahedron Lett. 25:45114514.

Fourrey, J.-L. and Varenne, J. 1985. Introduction of a nonaqueous oxidation procedure in the phosphite triester route for oligonucleotide synthesis. Tetrahedron Lett. 26:1217-1220.

Fourrey, J.-L., Varenne, J., Fontaine, C., Guittet, E., and Yang, Z.W. 1987. A new method for the synthesis of branched ribonucleotides. Tetrahedron Lett. 28:1769-1772.

Froehler, B. and Matteucci, M.D. 1983. Substituted 5-phenyltetrazoles: Improved activators of deoxynucleoside phosphoramidites in deoxyoligonucleotide synthesis. Tetrahedron Lett. 24:3171-3174.

Gaytán, P., Yañez, J., Sánchez, F., Mackie, H., and Soberón, X. 1998. Combination of DMTmononucleotide and Fmoc-trinucleotide phosphoramidites in oligonucleotide synthesis affords an automatable codon-level mutagenesis method. Chem. Biol. 5:519-527.

Gryaznov, S.M. and Letsinger, R.L. 1991. Synthesis of oligonucleotides via monomers with unprotected bases. J. Am. Chem. Soc. 113:5876-5877. 
Gryaznov, S.M. and Letsinger, R.L. 1992. Selective O-phosphitilation with nucleoside phosphoramidite reagents. Nucl. Acids Res. 20:18791882.

Guo, M., Yu, D., Iyer, R.P., and Agrawal, S. 1998. Solid-phase stereoselective synthesis of $2^{\prime}-O$ methyl oligoribonucleoside phosphorothioates using nucleoside oxazaphospholidines. Bioorg. Med. Chem. Lett. 8:2539-2544.

Hayakawa, Y. and Kataoka, M. 1998. Facile synthesis of oligodeoxyribonucleotides via the phosphoramidite method without nucleoside base protection. J. Am. Chem. Soc. 120:12395-12401.

Hayakawa, Y., Uchiyama, M., and Noyori, R. 1986. Nonaqueous oxidation of nucleoside phosphites to the phosphates. Tetrahedron Lett. 27:41914194.

Hayakawa, Y., Kataoka, M., and Noyori, R. 1996. Benzimidazolium triflate as an efficient promoter for nucleotide synthesis via the phosphoramidite method. J. Org. Chem. 61:79967997.

Hering, G., Stöcklein-Schneiderwind, R., Ugi, I., Pathak, T., Balgobin, N., and Chattopadhyaya, J. 1985. Preparation and properties of chloroN,N-dialkylamino-2,2,2-trichloroethoxy- and chloro-N,N-dialkylamino-2,2,2-trichloro-1,1dimethylethoxyphosphines and their deoxynucleoside phosphiteamidates. Nucleosides $\mathrm{Nu}$ cleotides 4:169-171.

Iyer, R.P., Phillips, L.R., Egan, W., Regan, J.B., and Beaucage, S.L. 1990. The automated synthesis of sulfur-containing oligodeoxyribonucleotides using 3H-1,2-benzodithiol-3-one 1,1-dioxide as a sulfur-transfer reagent. J. Org. Chem. 55:46934698.

Iyer, R.P., Yu, D., Habus, I., Ho, N.-H., Johnson, S., Devlin, T., Jiang, Z., Zhou, W., Xie, J., and Agrawal, S. 1997. N-Pent-4-enoyl (PNT) group as a universal nucleobase protector: Applications in the rapid and facile synthesis of oligonucleotides, analogs, and conjugates. Tetrahedron 53:2731-2750.

Iyer, R.P., Guo, M.-J., Yu, D., and Agrawal, S. 1998. Solid-phase stereoselective synthesis of oligonucleoside phosphorothioates: The nucleoside bicyclic oxazaphospholidines as novel synthons. Tetrahedron Lett. 39:2491-2494.

Iyer, R.P., Roland, A., Zhou, W., and Ghosh, K. 1999. Modified oligonucleotides-Synthesis, properties and applications. Curr. Opin. Mol. Ther. 1:344-358.

Jørgensen, P.N., Stein, P.C., and Wengel, J. 1994. Synthesis of $3^{\prime}-C$-(hydroxymethyl) thymidine: Introduction of a novel class of dexoynucleosides and oligodeoxynucleotides. J. Am. Chem. Soc. 116:2231-2232.

Josephson, S., Lagerholm, E., and Palm, G. 1984. Automatic synthesis of oligodeoxynucleotides and mixed oligodeoxynucleotides using the phosphoramidite method. Acta Chem. Scand. B38:539-545.
Kamer, P.C.J., Roelen, H.C.P.F., van den Elst, H., van der Marel, G.A., and van Boom, J.H. 1989. An efficient approach toward the synthesis of phosphorothioate diesters via the Schönberg reaction. Tetrahedron Lett. 30:6757-6760.

Kayushin, A.L., Korosteleva, M.D., Miroshnikov, A.I., Kosch, W., Zubov, D., and Piel, N. 1996. A convenient approach to the synthesis of trinucleotide phosphoramidites-Synthons for the generation of oligonucleotide/peptide libraries. Nucl. Acids Res. 24:3748-3755.

Khorana, H.G. 1968. Nucleic acid synthesis. Pure Appl. Chem. 17:349-381.

Kierzek, R., Rozek, M., and Markiewicz, W.T. 1987. Some steric aspects of synthesis of oligoribonucleotides by phosphoramidite approach on solid support. Nucl. Acids Res. Symp. Ser. No. 18:201204.

Kofoed, T. and Caruthers, M.H. 1996. Synthesis of 5 '-phosphonate linked thymidine deoxyoligonucleotides. Tetrahedron Lett. 37:6457-6460.

Krotz, A.H., Klopchin, P.G., Walker, K.L., Srivatsa, G.S., Cole, D.L., and Ravikumar, V.T. 1997a. On the formation of longmers in phosphorothioate oligodeoxyribonucleotide synthesis. Tetrahedron Lett. 38:3875-3878.

Krotz, A.H., Klopchin, P., Cole, D.L., and Ravikumar, V.T. 1997b. Improved purity profile of phosphorothioate oligonucleotides through the use of dimeric phosphoramidite synthons. Nucleosides Nucleotides 16:1637-1640.

Kuijpers, W.H.A., Huskens, J., and van Boeckel, C.A.A. 1990. The 2-(acetoxymethyl)benzoyl (AMB) group as a new base-protecting group, designed for the protection of (phosphate) modified oligonucleotides. Tetrahedron Lett. 31:6729-6732.

Kumar, G. and Poonian, M.S. 1984. Improvements in oligodeoxyribonucleotide synthesis: Methyl $\mathrm{N}, \mathrm{N}$-dialkylphosphoramidite dimer units for solid support phosphite methodology. J. Org. Chem. 49:4905-4912.

Lee, H.-J. and Moon, S.-H. 1984. Bis-(N,N-dialkylamino)-alkoxyphosphines as a new class of phosphite coupling agent for the synthesis of oligonucleotides. Chem. Lett. 1229-1232.

Letsinger, R.L. and Lunsford, W.B. 1976. Synthesis of thymidine oligonucleotides by phosphite triester intermediates. J. Am. Chem. Soc. 98:36553661 .

Letsinger, R.L. and Mahadevan, V. 1966. Stepwise synthesis of oligodeoxyribonucleotides on an insoluble polymer support. J. Am. Chem. Soc. 88:5319-5324.

Letsinger, R.L. and Ogilvie, K.K. 1969. Synthesis of oligothymidylates via phosphotriester intermediates. J. Am. Chem. Soc. 91:3350-3355.

Lyttle, M.H., Napolitano, E.W., Calio, B.L., and Kauvar, L.M. 1995. Mutagenesis using trinucleotide $\beta$-cyanoethyl phosphoramidites. BioTechniques 19:274-280.
Synthesis of Unmodified Oligonucleotides 
Mathis, R., Lafaille, L., and Burgada, R. 1974. Fréquence d'absorption de la liaison $\mathrm{P}-\mathrm{N}$ dans des composés du phosphore tricoordonné. Spectrochim. Acta, Part A 30:357-370.

Matteucci, M.D. and Caruthers, M.H. 1981. Synthesis of deoxyoligonucleotides on a polymer support. J. Am. Chem. Soc. 103:3185-3191.

McBride, L.J. and Caruthers, M.H. 1983. An investigation of several deoxynucleoside phosphoramidites useful for synthesizing deoxyoligonucleotides. Tetrahedron Lett. 24:245-248.

McBride, L.J., Kierzek, R., Beaucage, S.L., and Caruthers, M.H. 1986. Amidine protecting groups for oligonucleotide synthesis. J. Am. Chem. Soc. 108:2040-2048.

Montserrat, F.X., Cerandas, A., Eritja, R., and Pedroso, E. 1994. Criteria for the economic large scale solid-phase synthesis of oligonucleotides. Tetrahedron 50:2617-2622.

Moore, M.F. and Beaucage, S.L. 1985. Conceptual basis of the selective activation of bis(dialkylamino)methoxyphosphines by weak acids and its application toward the preparation of deoxynucleoside phosphoramidites in situ. J. Org. Chem. 50:2019-2025.

Mullah, B., Andrus, A., Zhao, H., and Jones, R.A. 1995. Oxidative conversion of $N$-dimethylformamidine nucleosides to $N$-cyano nucleosides. Tetrahedron Lett. 36:4373-4376.

Natt, F. and Häner, R. 1997. Lipocap: A lipophilic phosphoramidite-based capping reagent. Tetrahedron 53:9629-9636.

Neuner, P., Cortese, R., and Monaci, P. 1998. Codon-based mutagenesis using dimer-phosphoramidites. Nucl. Acids Res. 26:1223-1227.

Nielsen, J. and Caruthers, M.H. 1988. Directed Arbuzov-type reactions of 2-cyano-1,1-dimethylethyl deoxynucleoside phosphites. J. Am. Chem. Soc. 110:6275-6276.

Ono, A., Matsuda, A., Zhao, J., and Santi, D.V. 1995. The synthesis of blocked triplet-phosphoramidites and their use in mutagenesis. Nucl. Acids Res. 23:4677-4682.

Polushin, N.N. 1996. Synthesis of functionally modified oligonucleotides from methoxyoxalamido precursors. Tetrahedron Lett. 37:32313234.

Pon, R.T., Usman, N., Damha, M.J., and Ogilvie, K.K. 1986. Prevention of guanine modification and chain cleavage during the solid phase synthesis of oligonucleotides using phosphoramidite derivatives. Nucl. Acids Res. 14:6453-6470.

Pon, R.T. 1987. Enhanced coupling efficiency using 4-dimethylamino pyridine (DMAP) and either tetrazole, 5-(o-nitrophenyl) tetrazole or 5-( $p$ nitrophenyl) tetrazole in the solid phase synthesis of oligoribonucleotides by the phosphoramidite procedure. Tetrahedron Lett. 28: 3643-3646.
Rao, M.V. and Macfarlane, K. 1994. Solid phase synthesis of phosphorothioate oligonucleotides using benzyltriethylammonium tetrathiomolybdate as a rapid sulfur transfer reagent. Tetrahedron Lett. 35:6741-6744.

Rao, M.V., Reese, C.B., and Zhengyun, Z. 1992. Dibenzoyl tetrasulphide-A rapid sulphur transfer agent in the synthesis of phosphorothioate analogues of oligonucleotides. Tetrahedron Lett. 33:4839-4842.

Reddy, M.P., Hanna, N.B., and Farooqui, F. 1994. Fast cleavage and deprotection of oligonucleotides. Tetrahedron Lett. 35:4311-4314.

Reddy, M.P., Hanna, N.B., and Farooqui, F. 1997. Ultrafast cleavage and deprotection of oligonucleotides-Synthesis and use of $\mathrm{C}^{\mathrm{Ac}}$ derivatives. Nucleosides Nucleotides 16:1589-1598.

Regan, J.B., Phillips, L.R., and Beaucage, S.L. 1992. Large-scale preparation of the sulfurtransfer reagent $3 \mathrm{H}$-1,2-benzodithiol-3-one 1,1dioxide. Org. Prep. Proc. Int. 24:488-492.

Roelen, H.C.P.F., Kamer, P.C.J., van den Elst, H., van der Marel, G.A., and van Boom, J.H. 1991. A study on the use of phenylacetyl disulfide in the solid-phase synthesis of oligodeoxynucleoside phosphorothioates. Recl. Trav. Chim. Pays-Bas 110:325-331.

Sanghvi, Y.S. and Cook, P.D. 1994. Carbohydrates: Synthetic methods and applications in antisense therapeutics. In ACS Symposium Series 580Carbohydrate Modifications in Antisense Research (Y.S. Sanghvi and P.D. Cook, eds.) pp. 1-22. American Chemical Society, Washington, D.C.

Schulhof, J.C., Molko, D., and Teoule, R. 1987. The final deprotection step in oligonucleotide synthesis is reduced to a mild and rapid ammonia treatment by using labile base-protecting groups. Nucl. Acids Res. 15:397-416.

Schwartz, M.E., Breaker, R.R., Asteriadis, G.T., deBear, J.S., and Gough, G.R. 1992. Rapid synthesis of oligoribonucleotides using $2^{\prime}-O$ - $(o-n i-$ trobenzyloxymethyl)-protected monomers. Bioorg. Med. Chem. Lett. 2:1019-1024.

Sekine, M., Masuda, N., and Hata, T. 1986. Synthesis of oligodeoxyribonucleotides involving a rapid procedure for the removal of base-protecting groups by use of the $4,4^{\prime}, 4^{\prime \prime}$-tris(benzoyloxy)trityl (TBTr) group. Bull. Chem. Soc. Jpn. 59:1781-1789.

Seliger, H. and Gupta, K.C. 1985. Three-phase synthesis of oligonucleotides. Angew. Chem. Int. Ed. Engl. 24:685-687.

Sinha, N.D., Biernat, J., McManus, J., and Köster, H. 1984. Polymer support oligonucleotide synthesis XVIII: Use of $\beta$-cyanoethyl- $N, N$-dialkylamino-/ $N$-morpholino phosphoramidite of deoxynucleosides for the synthesis of DNA fragments simplifying deprotection and isolation of the final product. Nucl. Acids Res. 12:45394557. 
Sinha, N.D., Davis, P., Usman, N., Pérez, J., Hodge, R., Kremsky, J., and Casale, R. 1993. Labile exocyclic amine protection of nucleosides in DNA, RNA and oligonucleotide analog synthesis facilitating $N$-deacylation, minimizing depurination and chain degradation. Biochimie 75:1323.

Sondek, J. and Shortle, D. 1992. A general strategy for random insertion and substitution mutagenesis: Substoichiometric coupling of trinucleotide phosphoramidites. Proc. Natl. Acad. Sci. U.S.A. 89:3581-3585.

Stec, W.J. and Zon, G. 1984. Stereochemical studies of the formation of chiral internucleotide linkages by phosphoramidite coupling in the synthesis of oligodeoxyribonucleotides. Tetrahedron Lett. 25:5279-5282.

Stec, W.J., Uznanski, B., Wilk, A., Hirschbein, B.L., Fearon, K.L., and Bergot, B.J. 1993. Bis( O,O-diisopropoxy phosphinothioyl) disulfide-A highly efficient sulfurizing reagent for cost-effective synthesis of oligo(nucleoside phosphorothioate)s. Tetrahedron Lett. 34:5317-5320.

Tanaka, T. and Letsinger, R.L. 1982. Syringe method for the stepwise chemical synthesis of oligonucleotides. Nucl. Acids Res. 10:32493260 .

Tener, G.M. 1961. 2-Cyanoethyl phosphate and its use in the synthesis of phosphate esters. J. Am. Chem. Soc. 83:159-168.

Ugi, I., Jacob, P., Landgraf, B., Rupp, C., Lemmen, P., and Verfürth, U. 1988. Phosphite oxidation and the preparation of five-membered cyclic phosphorylating reagents via the phosphites. $\mathrm{Nu}$ cleosides Nucleotides 7:605-608.

Usman, N., Pon, R.T., and Ogilvie, K.K. 1985. Preparation of ribonucleoside 3'-O-phosphoramidites and their application to the automated solid phase synthesis of oligonucleotides. Tetrahedron Lett. 26:4567-4570.

Uznanski, B., Grajkowski, A., and Wilk, A. 1989. The isopropoxyacetic group for convenient base protection during solid-support synthesis of oligodeoxyribonucleotides and their triester analogs. Nucl. Acids Res. 17:4863-4871.

Vargeese, C., Carter, J., Yegge, J., Krivjansky, S., Settle, A., Kropp, E., Peterson, K., and Pieken, W. 1998. Efficient activation of nucleoside phosphoramidites with 4,5-dicyanoimidazole during oligonucleotide synthesis. Nucl. Acids Res. 26:1046-1050.

Virnekäs, B., Ge, L., Plückthun, A., Schneider, K.C., Wellnhofer, G., and Moroney, S.E. 1994. Trinucleotide phosphoramidites: ideal reagents for the synthesis of mixed oligonucleotides for random mutagenesis. Nucl. Acids Res. 22:5600-5607.

Vu, H. and Hirschbein, B.L. 1991. Internucleotide phosphite sulfurization with tetraethylthiuram disulfide. Phosphorothioate oligonucleotide synthesis via phosphoramidite chemistry. Tetrahedron Lett. 32:3005-3008.
Vu, H., McCollum, C., Jacobson, K., Theisen, P., Vinayak, R., Spiess, E., and Andrus, A. 1990. Fast oligonucleotide deprotection phosphoramidite chemistry for DNA synthesis. Tetrahedron Lett. 31:7269-7272.

Weber, H. and Khorana, H.G. 1972. CIV. Total synthesis of the structural gene for an alanine transfer ribonucleic acid from yeast. Chemical synthesis of an icosadeoxynucleotide corresponding to the nucleotide sequence 21 to $40 . J$. Mol. Biol. 72:219-249.

Weiss, P. 1998. TOM-protecting group-A major improvement in RNA synthesis. The Glen Report 11:2-4.

Wilk, A., Srinivasachar, K., and Beaucage, S.L. 1997. $N$-Trifluoroacetylamino alcohols as phosphodiester protecting groups in the synthesis of oligodeoxyribonucleotides. J. Org. Chem. 62:6712-6713.

Wincott, F., DiRenzo, A., Shaffer, C., Grimm, S., Tracz, D., Workman, C., Sweedler, D., Gonzalez, C., Scaringe, S., and Usman, N. 1995. Synthesis, deprotection, analysis and purification of RNA and ribosymes. Nucl. Acids Res. 23:2677-2684.

Wolter, A., Biernat, J., and Köster, H. 1986. Polymer support oligonucleotide synthesis XX: Synthesis of a henhectacosa deoxynucleotide by use of a dimeric phosphoramidite synthon. Nucleosides Nucleotides 5:65-77.

Wu, X. and Pitsch, S. 1998. Synthesis and pairing properties of oligoribonucleotide analogues containing a metal-binding site attached to $\beta$-D-allofuranosyl cytosine. Nucl. Acids Res. 26:43154323.

Xin, Z. and Just, G. 1996. Diastereoselective synthesis of phosphite triesters. Tetrahedron Lett. 37:969-972.

Xu, Q., Barany, G., Hammer, R.P., Musier-Forsyth, K. 1996. Efficient introduction of phosphorothioates into RNA oligonucleotides by 3ethoxy-1,2,4-dithiazoline-5-one (EDITH). Nucl. Acids Res. 24:3643-3644.

Yamana, K., Nishijima, Y., Oka, A., Nakano, H., Sangen, O., Ozaki, H., and Shimidzu, T. 1989. A simple preparation of $5^{\prime}$ - $O$-dimethoxytrityl deoxyribonucleoside 3'-O-phosphor-bisdiethylamidites as useful intermediates in the synthesis of oligodeoxyribonucleotides and their phosphorodiethylamidate analogs on a solid support. Tetrahedron 45:4135-4140.

Yu, D., Tang, J.-Y., Iyer, R.P., and Agrawal, S. 1994. Diethoxy $N, N$-diisopropyl phosphoramidite as an improved capping reagent in the synthesis of oligonucleotides using phosphoramidite chemistry. Tetrahedron Lett. 35:8565-8568.

Zehl, A., Starke, A., Cech, D., Hartsch, T., Merkl, R., and Fritz, H.-J. 1996. Efficient and flexible access to fully protected trinucleotides suitable for DNA synthesis by automated phosphoramidite chemistry. Chem. Commun. 26772678.
Synthesis of Unmodified Oligonucleotides

Current Protocols in Nucleic Acid Chemistry 
Zemlicka, J. and Holy, A. 1967. Preparation of $N$-dimethylaminomethylene derivatives-A new method of a selective substitution of nucleoside amino groups. Coll. Czech. Chem. Commun. 32:3159-3168.

Zhang, Z., Nichols, A., Alsbeti, M., Tang, J.X., and Tang, J.Y. 1998. Solid phase synthesis of oligonucleotide phosphorothioate analogues using bis(ethoxythiocarbonyl)tetrasulfide as a new sulfur-transfer reagent. Tetrahedron Lett. 39:24672470.

Zhang, Z., Nichols, A., Tang, J.X., Han, Y., and Tang, J.Y. 1999. Solid phase synthesis of oligonucleotide phosphorothioate analogues using 3methyl-1,2,4-dithiazolin-5-one (MEDITH) as a new sulfur-transfer reagent. Tetrahedron Lett. 40:2095-2098.

Zhao, Z. and Caruthers, M.H. 1996. Synthesis and preliminary biochemical studies with $5^{\prime}$-deoxy5 '-methylidyne phosphonate linked thymidine oligonucleotides. Tetrahedron Lett. 37:62396242.
Zon, G., Gallo, K.A., Samson, C.J., Shao, K., Summers, M.F., and Byrd, R.A. 1985. Analytical studies of 'mixed sequence' oligodeoxyribonucleotides synthesized by competitive coupling of either methyl- or $\beta$-cyanoethyl- $N, N$-diisopropylamino phosphoramidite reagents, including $2^{\prime}$ deoxyinosine. Nucl. Acids Res. 13:8181-8196.

Contributed by Serge L. Beaucage

Center for Biologics Evaluation and Research Food and Drug Administration

Bethesda, Maryland

Marvin H. Caruthers

University of Colorado

Boulder, Colorado
Oligodeoxyribonucleotide Synthesis Using the Phosphoramidite Method 